\title{
Global weak solutions for a modified two-component Camassa-Holm equation
}

\author{
Chunxia Guan, Zhaoyang Yin * \\ Department of Mathematics, Sun Yat-sen University, 510275 Guangzhou, China \\ Received 23 September 2010; accepted 26 April 2011 \\ Available online 7 May 2011
}

\begin{abstract}
We obtain the existence of global-in-time weak solutions for the Cauchy problem of a modified two-component Camassa-Holm equation. The global weak solution is obtained as a limit of viscous approximation. The key elements in our analysis are the Helly theorem and some a priori one-sided supernorm and space-time higher integrability estimates on the first-order derivatives of approximation solutions.
\end{abstract}

(c) 2011 Elsevier Masson SAS. All rights reserved.

\section{Résumé}

Nous obtenons l'existence globale en temps de solutions faibles pour le problème de Cauchy d'une équation modifiée CamassaHolm à deux composantes. La solution faible globale est obtenue comme une limite de par approximation visqueuse. Les éléments clé dans notre analyse sont le théorème de Helly et certaines estimations a priori de supernorme d'un seul côté et d'intégrabilité dans l'espace-temps des dérivées premières des solutions approchées.

(C) 2011 Elsevier Masson SAS. All rights reserved.

MSC: $35 \mathrm{G} 25 ; 35 \mathrm{~L} 05$

Keywords: A modified two-component Camassa-Holm equation; Well-posedness; Blow-up scenario; Strong solution; Global weak solution

\section{Introduction}

In this paper we consider the Cauchy problem of the following modified two-component Camassa-Holm equation:

$$
\begin{cases}m_{t}+u m_{x}+2 m u_{x}=-\rho \bar{\rho}_{x}, & t>0, x \in \mathbb{R}, \\ \rho_{t}+(\rho u)_{x}=0, & t>0, x \in \mathbb{R}, \\ m(0, x)=m_{0}(x), & x \in \mathbb{R}, \\ \rho(0, x)=\rho_{0}(x), & x \in \mathbb{R},\end{cases}
$$

where $m=u-u_{x x}$ and $\rho=\left(1-\partial_{x}^{2}\right)\left(\bar{\rho}-\bar{\rho}_{0}\right)$.

\footnotetext{
* Corresponding author.

E-mail addresses: guanchunxia123@163.com (C. Guan), mcsyzy@mail.sysu.edu.cn (Z. Yin).
} 
The Camassa-Holm equation has been recently extended to a two-component integrable system $(\mathrm{CH} 2)$ by combing its integrability property with compressibility, or free-surface elevation dynamics in its shallow-water interpretation [7,25]. Eq. (1.1) was recently introduced by Holm et al. in [40]. The modified two-component Camassa-Holm equation (MCH2) is written in terms of velocity $u$ and locally averaged density $\bar{\rho}$ (or depth, in the shallow-water interpretation) and $\bar{\rho}_{0}$ is taken to be constant. $\mathrm{MCH} 2$ is defined as geodesic motion on the semidirect product Lie group with respect to a certain metric and is given as a set of Euler-Poincaré equations on the dual of the corresponding Lie algebra [39].

For $\rho \equiv 0$, Eq. (1.1) becomes the Camassa-Holm equation, modeling the unidirectional propagation of shallow water waves over a flat bottom. Here $u(t, x)$ stands for the fluid velocity at time $t$ in the spatial $x$ direction [5,14]. The Camassa-Holm equation is also a model for the propagation axially symmetric waves in hyperelastic rods [27]. It has a bi-Hamiltonian structure $[9,32]$ and is completely integrable [5,12]. Its traveling waves (periodic as well as solitary) are peaked [6], capturing thus the shape of solitary wave solutions to the governing equations for water waves $[13,19]$. The orbital stability of the peaked solutions is proved in [24]. The explicit interaction of the peaked solutions is given in [2].

The Cauchy problem for the Camassa-Holm equation has been studied extensively $[16,28,47,52]$. It has been shown that this equation is locally well posed $[15,28,47,52]$ for initial data $u_{0} \in H^{s}(\mathbb{R}), s>\frac{3}{2}$. More interesting, it has global strong solutions [11,15] and also finite time blow-up solutions [11,15-17,28,47]. On the other hand, it has global weak solutions in $H^{1}(\mathbb{R})[3,4,8,23,36-38,51]$. Recently, it was claimed in [46] that the Camassa-Holm equation might be relevant to the modeling of tsunamis (see also the discussion in [20]).

The advantage of the Camassa-Holm equation in comparison with the KdV equation lies in the fact that while both KdV and Camassa-Holm equations are completely integrable Hamiltonian systems [10,22,30]; while the inverse scattering approach was obtained in [18] (see also [33]), in addition to KdV the Camassa-Holm equation has peaked solitons and models wave breaking $[6,16]$ (by wave breaking we understand that the wave remains bounded while its slop becomes unbounded in finite time [50]).

Recently, two types of 2-component Camassa-Holm equations have been studied in [7,25,31,34]. These works have established the local well-posedness [25,31], derived precise blow-up scenarios [31], and proved the existence of strong solutions which blow up in finite time [25,31,34]. More recently, the Cauchy problem of Eq. (1.1) has been studied in [35].

However, the existence of global weak solutions to Eq. (1.1) has not been discussed yet. Our aim of this paper is to prove the existence of global week solutions to Eq. (1.1) provided the initial data satisfying some certain conditions. We hope that our result sheds some light on important physical phenomena of Eq. (1.1) such as wave breaking. Up to now, we have no uniqueness result for the obtained global weak solutions to Eq. (1.1). This problem will be discussed later on. Note that no global existence results for strong solutions to Eq. (1.1) are available so far. Thus, we have to use the viscous approximation method to prove the existence of global weak solution to Eq. (1.1).

We now provide the framework in which we shall reformulate problem (1.1). With $m=u-u_{x x}, \rho=\gamma-\gamma_{x x}$ and $\gamma=\bar{\rho}-\bar{\rho}_{0}$, we can rewrite Eq. (1.1) as follows:

$$
\begin{cases}m_{t}+m_{x} u+2 m u_{x}=-\rho \gamma_{x}, & t>0, x \in \mathbb{R}, \\ \rho_{t}+(u \rho)_{x}=0, & t>0, x \in \mathbb{R}, \\ m(0, x)=u_{0}(x)-u_{0, x x}(x), & x \in \mathbb{R}, \\ \rho(0, x)=\gamma_{0}-\gamma_{0, x x}, & x \in \mathbb{R} .\end{cases}
$$

Note that if $p(x):=\frac{1}{2} e^{-|x|}, x \in \mathbb{R}$, then $\left(1-\partial_{x}^{2}\right)^{-1} f=p * f$ for all $f \in L^{2}(\mathbb{R}), p * m=u$ and $p * \rho=\gamma$. Here we denote by $*$ the convolution. Using this identity, we can rewrite Eq. (1.2) as follows:

$$
\begin{cases}u_{t}+u u_{x}=-\partial_{x} p *\left(u^{2}+\frac{1}{2} u_{x}^{2}+\frac{1}{2} \gamma^{2}-\frac{1}{2} \gamma_{x}^{2}\right), & t>0, x \in \mathbb{R}, \\ \gamma_{t}+u \gamma_{x}=-p *\left(\left(u_{x} \gamma_{x}\right)_{x}+u_{x} \gamma\right), & t>0, x \in \mathbb{R}, \\ u(0, x)=u_{0}(x), & x \in \mathbb{R}, \\ \gamma(0, x)=\gamma_{0}(x), & x \in \mathbb{R} .\end{cases}
$$


The main result of this paper is to give the existence of a globe-in-time weak solution $z=\left(\begin{array}{l}u \\ \gamma\end{array}\right)$ to the Cauchy problem (1.1) with the initial data $z_{0}=\left(\begin{array}{c}u_{0} \\ \gamma_{0}\end{array}\right) \in H^{1}(\mathbb{R}) \times H^{1}(\mathbb{R})$. Before giving the precise statement of the main result, we first introduce the definition of a weak solution to the Cauchy problem (1.1).

Definition 1.1. $z=\left(\begin{array}{l}u \\ \gamma\end{array}\right)$ is said to be an admissible weak solution to the Cauchy problem (1.1) if

$$
z(t, x) \in L^{\infty}\left((0, \infty) ; H^{1}(\mathbb{R}) \times H^{1}(\mathbb{R})\right)
$$

satisfies Eq. (1.3) and $z(t, \cdot) \rightarrow z_{0}$ as $t \rightarrow 0^{+}$in the sense of distributions, and for any $t>0$,

$$
\|z(t, \cdot)\|_{H^{1}(\mathbb{R}) \times H^{1}(\mathbb{R})} \leqslant\left\|z_{0}\right\|_{H^{1}(\mathbb{R}) \times H^{1}(\mathbb{R})} .
$$

Notation. In our paper, the space of all Radon measures on $\mathbb{R}$ is denoted by $\mathcal{M}(\mathbb{R})$ and $\mathcal{M}^{+}(\mathbb{R})$ denotes the set of all positive Radon measures.

The main result of this paper can be stated as follows:

Theorem 1.1. Let $z_{0}=\left(\begin{array}{c}u_{0} \\ \gamma_{0}\end{array}\right) \in H^{1}(\mathbb{R}) \times H^{1}(\mathbb{R})$. If $\rho_{0}=\gamma_{0}-\gamma_{0, x x} \in \mathcal{M}^{+}(\mathbb{R})$, then Eq. (1.1) has an admissible weak solution in the sense of Definition 1.1.

Our paper is organized as follows. In Section 2, we give the approximate solutions $z_{\epsilon}=\left(\begin{array}{l}u_{\epsilon} \\ \gamma_{\epsilon}\end{array}\right)$ to Eq. (1.1) and derive the basic energy estimate on $z_{\epsilon}$. In Section 3, the crucial uniform a priori one-side supernorm estimate and local space-time higher integrability estimate for $u_{\epsilon}$ are derived. Moreover, using Helly theorem we get that $\partial_{x} \gamma_{\epsilon}$ is convergent almost everywhere on $\mathbb{R}_{+} \times \mathbb{R}$. Finally, the strong convergence of $\partial_{x} u_{\epsilon}$ in $L_{l o c}^{2}\left(\mathbb{R}_{+} \times \mathbb{R}\right)$ is carried out and we conclude the proof of the main result in Section 4.

\section{Viscous approximate solutions}

In the section, we construct the approximate solution sequence $z_{\epsilon}=z_{\epsilon}(t, x)=\left(\begin{array}{l}u_{\epsilon}(t, x) \\ \gamma_{\epsilon}(t, x)\end{array}\right)$ as solutions to the viscous problem of Eq. (1.1), i.e.,

$$
\begin{cases}u_{t}+u u_{x}+\partial_{x} p *\left(u^{2}+\frac{1}{2} u_{x}^{2}+\frac{1}{2} \gamma^{2}-\frac{1}{2} \gamma_{x}^{2}\right)=\epsilon u_{x x}, & t>0, x \in \mathbb{R}, \\ \gamma_{t}+u \gamma_{x}+\partial_{x} p *\left(u_{x} \gamma_{x}\right)+p *\left(u_{x} \gamma\right)=0, & t>0, x \in \mathbb{R}, \\ u(0, x)=u_{\epsilon, 0}(x), & x \in \mathbb{R}, \\ \gamma(0, x)=\gamma_{\epsilon, 0}(x), & x \in \mathbb{R},\end{cases}
$$

or the equivalent form:

$$
\begin{cases}u_{t}+u u_{x}=-\partial_{x}\left(1-\partial_{x}^{2}\right)^{-1}\left(u^{2}+\frac{1}{2} u_{x}^{2}+\frac{1}{2} \gamma^{2}-\frac{1}{2} \gamma_{x}^{2}\right)+\epsilon u_{x x}, & t>0, x \in \mathbb{R}, \\ \gamma_{t}+u \gamma_{x}=-\left(1-\partial_{x}^{2}\right)^{-1}\left(\left(u_{x} \gamma_{x}\right)_{x}+u_{x} \gamma\right), & t>0, x \in \mathbb{R}, \\ u(0, x)=u_{\epsilon, 0}(x), & x \in \mathbb{R}, \\ \gamma(0, x)=\gamma_{\epsilon, 0}(x), & x \in \mathbb{R},\end{cases}
$$

here $u_{\epsilon, 0}(x)=\left(\phi_{\epsilon} * u_{0}\right)(x), \gamma_{\epsilon, 0}(x)=\left(\phi_{\epsilon} * \gamma_{0}\right)(x) \in H^{s}(\mathbb{R}), s>2$, and

$$
\phi_{\epsilon}(x):=\left(\int_{\mathbb{R}} \phi(\xi) d \xi\right)^{-1} \frac{1}{\epsilon} \phi\left(\frac{x}{\epsilon}\right), \quad x \in \mathbb{R}, \epsilon>0,
$$

where $\phi \in C_{c}^{\infty}(\mathbb{R})$ is defined by

$$
\phi(x)= \begin{cases}e^{1 /\left(x^{2}-1\right)}, & |x|<1, \\ 0, & |x| \geqslant 1 .\end{cases}
$$


Then, we have

$$
\left\|u_{\epsilon, 0}\right\|_{H^{1}(\mathbb{R})} \leqslant\left\|u_{0}\right\|_{H^{1}(\mathbb{R})}, \quad\left\|\gamma_{\epsilon, 0}\right\|_{H^{1}(\mathbb{R})} \leqslant\left\|\gamma_{0}\right\|_{H^{1}(\mathbb{R})},
$$

and

$$
u_{\epsilon, 0} \rightarrow u_{0}, \quad \gamma_{\epsilon, 0} \rightarrow \gamma_{0}, \quad \text { in } H^{1}(\mathbb{R}) .
$$

Theorem 2.1. Assume $z_{0}=\left(\begin{array}{c}u_{0} \\ \gamma_{0}\end{array}\right) \in H^{1}(\mathbb{R}) \times H^{1}(\mathbb{R})$ and $\rho_{0}=\gamma_{0}-\gamma_{0, x x} \in \mathcal{M}^{+}(\mathbb{R})$. Then there exists a unique solution $z_{\epsilon}=\left(\begin{array}{c}u_{\epsilon} \\ \gamma_{\epsilon}\end{array}\right) \in C\left(\mathbb{R}_{+} ; H^{s}(\mathbb{R}) \times H^{s}(\mathbb{R})\right) \cap C^{1}\left(\mathbb{R}_{+} ; L^{2}(\mathbb{R}) \times L^{2}(\mathbb{R})\right)$, $s>2$, to the Cauchy problem (2.1). Moreover, for each $t \geqslant 0$,

$$
\begin{aligned}
& \int_{\mathbb{R}}\left(u_{\epsilon}^{2}+\gamma_{\epsilon}^{2}+\left(\frac{\partial u_{\epsilon}}{\partial x}\right)^{2}+\left(\frac{\partial \gamma_{\epsilon}}{\partial x}\right)^{2}\right)(t, x) d x+2 \epsilon \int_{0}^{t} \int_{\mathbb{R}}\left(\left(\frac{\partial u_{\epsilon}}{\partial x}\right)^{2}+\left(\frac{\partial^{2} u_{\epsilon}}{\partial x^{2}}\right)^{2}\right)(s, x) d x d s \\
& \quad=\left\|z_{\epsilon, 0}\right\|_{H^{1}(\mathbb{R}) \times H^{1}(\mathbb{R})}^{2} \leqslant\left\|z_{0}\right\|_{H^{1}(\mathbb{R}) \times H^{1}(\mathbb{R})}^{2},
\end{aligned}
$$

or

$$
\left\|z_{\epsilon}(t, \cdot)\right\|_{H^{1}(\mathbb{R}) \times H^{1}(\mathbb{R})}^{2}+2 \epsilon \int_{0}^{t}\left\|\frac{\partial u_{\epsilon}}{\partial x}(s, \cdot)\right\|_{H^{1}(\mathbb{R})}^{2} d s=\left\|z_{\epsilon, 0}\right\|_{H^{1}(\mathbb{R}) \times H^{1}(\mathbb{R})}^{2} \leqslant\left\|z_{0}\right\|_{H^{1}(\mathbb{R}) \times H^{1}(\mathbb{R})}^{2} .
$$

Before proving Theorem 2.1, we recall some useful lemmas.

Lemma 2.1. (See [44].) Let $f \in H^{s}(\mathbb{R}), s>\frac{3}{2}$. Then

$$
\left\|\Lambda^{-r}\left[\Lambda^{r+t+1}, M_{f}\right] \Lambda^{-t}\right\|_{L^{2}(\mathbb{R})} \leqslant c\|f\|_{H^{s}(\mathbb{R})}, \quad|r|,|t| \leqslant s-1,
$$

where $M_{f}$ is the operator of multiplication by $f$ and $c$ is a positive constant depending only on $r, t$.

Lemma 2.2. (See [43].) Let $r$, $t$ be real numbers such that $-r<t \leqslant r$. Then

$$
\begin{aligned}
& \|f g\|_{H^{t}(\mathbb{R})} \leqslant c\|f\|_{H^{r}}\|g\|_{H^{t}(\mathbb{R})}, \quad \text { if } r>\frac{1}{2}, \\
& \|f g\|_{H^{t+r-\frac{1}{2}(\mathbb{R})}} \leqslant c\|f\|_{H^{r}(\mathbb{R})}\|g\|_{H^{t}(\mathbb{R})}, \quad \text { if } r<\frac{1}{2},
\end{aligned}
$$

where $c$ is a positive constant depending on $r, t$.

Lemma 2.3. (See [26].) If $X_{1}$ and $X_{2}$ are Banach spaces and $A_{i} \in G\left(X_{i}, 1, \beta\right), i=1,2$, then the operator

$$
A=\left(\begin{array}{cc}
A_{1} & 0 \\
0 & A_{2}
\end{array}\right) \in G\left(X_{1} \times X_{2}, 1, \beta\right)
$$

with $D(A)=D\left(A_{1}\right) \times D\left(A_{2}\right)$.

The strategy of the proof of Theorem 2.1 is rather routine. We use some lemmas to prove this theorem. For the convenience of presentation, we will omit the subscripts in $z_{\epsilon}, u_{\epsilon}, \gamma_{\epsilon}$ in the following proofs.

Lemma 2.4. Given $z_{0}=\left(\begin{array}{c}u_{0} \\ \gamma_{0}\end{array}\right) \in H^{s}(\mathbb{R}) \times H^{s}(\mathbb{R}), s>2$, there exist a maximal $T=T\left(\left\|z_{0}\right\|_{H^{s}(\mathbb{R}) \times H^{s}(\mathbb{R})}\right)>0$, and a unique solution $z=\left(\begin{array}{l}u \\ \gamma\end{array}\right)$ to Eq. (2.1) such that

$$
z=z\left(\cdot, z_{0}\right) \in C\left([0, T) ; H^{s}(\mathbb{R}) \times H^{s}(\mathbb{R})\right) .
$$

Proof. Let $A(z)=\left(\begin{array}{cc}u \partial_{x}-\epsilon \partial_{x x} & 0 \\ 0 & u \partial_{x}\end{array}\right)=\left(\begin{array}{cc}A_{1}(z)+A_{0} & 0 \\ 0 & A_{1}(z)\end{array}\right)$ and

$$
f(z)=\left(\begin{array}{c}
-\partial_{x}\left(1-\partial_{x}^{2}\right)^{-1}\left(u^{2}+\frac{1}{2} u_{x}^{2}+\frac{1}{2} \gamma^{2}-\frac{1}{2} \gamma_{x}^{2}\right) \\
-\left(1-\partial_{x}^{2}\right)^{-1}\left(\left(u_{x} \gamma_{x}\right)_{x}+u_{x} \gamma\right)
\end{array}\right) .
$$


Set $Y=H^{s}(\mathbb{R}) \times H^{s}(\mathbb{R}), X=L^{2}(\mathbb{R}) \times L^{2}(\mathbb{R}), \Lambda=\left(1-\partial_{x}^{2}\right)^{\frac{1}{2}}$ and $Q=\left(\begin{array}{cc}\Lambda^{s} & 0 \\ 0 & \Lambda^{s}\end{array}\right)$. Obviously, $Q$ is an isomorphism of $H^{s}(\mathbb{R}) \times H^{s}(\mathbb{R})$ onto $L^{2}(\mathbb{R}) \times L^{2}(\mathbb{R})$ and $\Lambda^{s}$ is an isomorphism of $H^{s}(\mathbb{R})$ onto $L^{2}(\mathbb{R})$. In order to prove Lemma 2.4, we use Kato's semigroup approach [43].

We know that $A_{1}(z) \in G\left(L^{2}(\mathbb{R}), 1, \beta_{0}(z)\right)$ in [52]. It is obvious that $A_{0}$ is a densely defined closed linear operator in $X$ and is self-adjoint. For every $u \in D\left(A_{0}\right)=H^{2}(\mathbb{R})$,

$$
\left(A_{0} u, u\right)_{L^{2}(\mathbb{R})}=-\epsilon\left(\partial_{x x} u, u\right)_{L^{2}(\mathbb{R})}=\epsilon\left\|\partial_{x} u\right\|_{L^{2}(\mathbb{R})}^{2} .
$$

By Corollary 4.4 in Chapter 1 of [49] we have that $-A_{0}$ is the infinitesimal generator of a $C_{0}$ semigroup of contractions on $L^{2}(\mathbb{R})$. By $A_{1}(z) \in G\left(L^{2}(\mathbb{R}), 1, \beta_{0}(z)\right)$, therefore $-A_{1}(z)-\beta I$ is dissipative for all $\beta \geqslant \beta_{0}(z)$. Moreover, for every $y \in D\left(A_{1}\right)$,

$$
\left\|-\left(A_{1}(z)+\beta I\right) y\right\|_{L^{2}(\mathbb{R})} \leqslant\|u\|_{L^{\infty}\left\|\partial_{x} y\right\|_{L^{2}(\mathbb{R})}+\beta\|y\|_{L^{2}(\mathbb{R})} .}
$$

Using integration by parts, for every $y \in H^{2}(\mathbb{R})$, we have

$$
\left\|\partial_{x} y\right\|_{L^{2}(\mathbb{R})}^{2} \leqslant\|y\|_{L^{2}(\mathbb{R})}\left\|\partial_{x x} y\right\|_{L^{2}(\mathbb{R})}
$$

and

$$
\left\|-\left(A_{1}(z)+\beta I\right) y\right\|_{L^{2}(\mathbb{R})} \leqslant \frac{\epsilon}{2}\left\|\partial_{x x} y\right\|_{L^{2}(\mathbb{R})}+C\|y\|_{L^{2}(\mathbb{R})}=\frac{1}{2}\left\|-A_{0} y\right\|_{L^{2}(\mathbb{R})}+C\|y\|_{L^{2}(\mathbb{R})} .
$$

By Corollary 3.3.3 in [49], $-A_{0}-A_{1}(z)-\beta I$ is the infinitesimal generator of a $C_{0}$ semigroup of contractions of $L^{2}(\mathbb{R})$ for every $\beta \geqslant \beta_{0}(z)$. Therefore, $A_{1}(z)+A_{0} \in G\left(L^{2}(\mathbb{R}), 1, \beta(z)\right)$. Then, in view of Lemma 2.3 and $A_{1}(z) \in$ $G\left(L^{2}(\mathbb{R}), 1, \beta(z)\right)$, we have $A(z)=\left(\begin{array}{cc}A_{1}(z)+A_{0} & 0 \\ 0 & A_{1}(z)\end{array}\right) \in G\left(L^{2}(\mathbb{R}) \times L^{2}(\mathbb{R}), 1, \beta(z)\right)$.

Now we prove that $A(y) \in L\left(H^{s}(\mathbb{R}) \times H^{s}(\mathbb{R}), L^{2}(\mathbb{R}) \times L^{2}(\mathbb{R})\right)$ for $y \in H^{s}(\mathbb{R}) \times H^{s}(\mathbb{R})$ and we have that

$$
\|(A(y)-A(z)) w\|_{L^{2}(\mathbb{R}) \times L^{2}(\mathbb{R})} \leqslant \mu_{1}\|y-z\|_{L^{2}(\mathbb{R}) \times L^{2}(\mathbb{R})}\|w\|_{H^{s}(\mathbb{R}) \times H^{s}(\mathbb{R})},
$$

for $y, z, w \in H^{s}(\mathbb{R}) \times H^{s}(\mathbb{R})$.

Indeed, by the definition of $A(z)$, we deduce that for all $z, w \in H^{s}(\mathbb{R}) \times H^{s}(\mathbb{R})$,

$$
\|A(z) w\|_{L^{2}(\mathbb{R}) \times L^{2}(\mathbb{R})} \leqslant\left(\|z\|_{H^{s}(\mathbb{R}) \times H^{s}(\mathbb{R})}+\epsilon\right)\|w\|_{H^{s}(\mathbb{R}) \times H^{s}(\mathbb{R})} .
$$

For all $y, z, w \in H^{s}(\mathbb{R}) \times H^{s}(\mathbb{R})$,

$$
\begin{aligned}
\|(A(y)-A(z)) w\|_{L^{2}(\mathbb{R}) \times L^{2}(\mathbb{R})} & \leqslant\left(\left\|\partial_{x} w_{1}\right\|_{L^{\infty}(\mathbb{R})}+\left\|\partial_{x} w_{2}\right\|_{L^{\infty}(\mathbb{R})}\right)\|y-z\|_{L^{2}(\mathbb{R}) \times L^{2}(\mathbb{R})} \\
& \leqslant\|y-z\|_{L^{2}(\mathbb{R}) \times L^{2}(\mathbb{R})}\|w\|_{H^{s}(\mathbb{R}) \times H^{s}(\mathbb{R}) .}
\end{aligned}
$$

Note that

$$
B(z) y=Q A(z) Q^{-1} y-A(z) y=\left(\begin{array}{cc}
{\left[\Lambda^{s}, u \partial_{x}\right] \Lambda^{-s} y_{1}} & 0 \\
0 & {\left[\Lambda^{s}, u \partial_{x}\right] \Lambda^{-s} y_{2}}
\end{array}\right) .
$$

Let $w \in L^{2}(\mathbb{R}) \times L^{2}(\mathbb{R})$ and $y, z \in H^{s}(\mathbb{R}) \times H^{s}(\mathbb{R})$. Then we have

$$
\begin{aligned}
\|(B(y) w-B(z) w)\|_{L^{2}(\mathbb{R}) \times L^{2}(\mathbb{R})} & \leqslant\left\|\left[\Lambda^{s},\left(y_{1}-u\right) \partial_{x}\right] \Lambda^{-s} w_{1}\right\|_{L^{2}(\mathbb{R})}+\left\|\left[\Lambda^{s},\left(y_{1}-u\right) \partial_{x}\right] \Lambda^{-s} w_{2}\right\|_{L^{2}(\mathbb{R})} \\
& \leqslant\left\|\left[\Lambda^{s}, y_{1}-u\right] \Lambda^{1-s}\right\|_{L\left(L^{2}(\mathbb{R})\right)}\left(\left\|\Lambda^{-1} \partial_{x} w_{1}\right\|_{L^{2}(\mathbb{R})}+\left\|\Lambda^{-1} \partial_{x} w_{2}\right\|_{L^{2}(\mathbb{R})}\right) \\
& \leqslant c\left\|y_{1}-u\right\|_{H^{s}(\mathbb{R})}\|w\|_{L^{2}(\mathbb{R}) \times L^{2}(\mathbb{R})} \leqslant c\|y-z\|_{H^{s}(\mathbb{R}) \times H^{s}(\mathbb{R})}\|w\|_{L^{2}(\mathbb{R}) \times L^{2}(\mathbb{R})},
\end{aligned}
$$

where we applied Lemma 2.1 with $r=0, t=s-1$ in view of $s>2$. Taking $y=0$ in the above inequality, we obtain that $B(z) \in L\left(L^{2}(\mathbb{R}) \times L^{2}(\mathbb{R})\right)$.

Finally, we have that (see [35])

$$
\|f(y)-f(z)\|_{H^{s}(\mathbb{R}) \times H^{s}(\mathbb{R})} \leqslant \mu_{3}\|y-z\|_{H^{s}(\mathbb{R}) \times H^{s}(\mathbb{R})}, \quad \forall y, z \in H^{s}(\mathbb{R}) \times H^{s}(\mathbb{R}) .
$$

Thus, we only need to verify that 


$$
\begin{aligned}
\|f(y)-f(z)\|_{L^{2}(\mathbb{R}) \times L^{2}(\mathbb{R})} & \left\|\partial_{x}\left(1-\partial_{x}^{2}\right)^{-1}\left(\left(y_{1}^{2}-u^{2}\right)+\frac{1}{2}\left(y_{1, x}^{2}-u_{x}^{2}\right)+\frac{1}{2}\left(y_{2}^{2}-\gamma^{2}\right)-\frac{1}{2}\left(y_{2, x}^{2}-\gamma_{x}^{2}\right)\right)\right\|_{L^{2}(\mathbb{R})} \\
& +\left\|\left(1-\partial_{x}^{2}\right)^{-1}\left(\left(y_{1, x} y_{2, x}-u_{x} \gamma_{x}\right)_{x}+y_{1, x} y_{2}-u_{x} \gamma\right)\right\|_{L^{2}(\mathbb{R})} \\
\leqslant & \left\|\left(y_{1}^{2}-u^{2}\right)+\frac{1}{2}\left(y_{1, x}^{2}-u_{x}^{2}\right)+\frac{1}{2}\left(y_{2}^{2}-\gamma^{2}\right)-\frac{1}{2}\left(y_{2, x}^{2}-\gamma_{x}^{2}\right)\right\|_{H^{-1}(\mathbb{R})} \\
& +\left\|\left(y_{1, x} y_{2, x}-u_{x} \gamma_{x}\right)_{x}+y_{1, x} y_{2}-u_{x} \gamma\right\|_{H^{-2}(\mathbb{R})} \\
\leqslant & \left\|y_{1}-u\right\|_{L^{2}(\mathbb{R})}\left\|y_{1}+u\right\|_{L^{\infty}(\mathbb{R})}+\frac{c}{2}\left\|y_{1, x}-u_{x}\right\|_{H^{-1}(\mathbb{R})}\left\|y_{1, x}+u_{x}\right\|_{H^{s-1}(\mathbb{R})} \\
& +\frac{1}{2}\left\|y_{2}-\gamma\right\|_{L^{2}(\mathbb{R})}\left\|y_{2}+\gamma\right\|_{L^{\infty}(\mathbb{R})}+\frac{c}{2}\left\|y_{2, x}-\gamma_{x}\right\|_{H^{-1}(\mathbb{R})}\left\|y_{2, x}+\gamma_{x}\right\|_{H^{s-1}(\mathbb{R})} \\
& +\left\|y_{1, x} y_{2, x}-y_{1, x} \gamma_{x}\right\|_{H^{-1}(\mathbb{R})}+\left\|y_{1, x} \gamma_{x}-u_{x} \gamma_{x}\right\|_{H^{-1}(\mathbb{R})} \\
& +\left\|y_{1, x} y_{2}-y_{1, x} \gamma\right\|_{H^{-1}(\mathbb{R})}+\left\|y_{1, x} \gamma-u_{x} \gamma\right\|_{H^{-1}(\mathbb{R})} \\
\leqslant & \left\|y_{1}-u\right\|_{L^{2}(\mathbb{R})}\left\|y_{1}+u\right\|_{L^{\infty}(\mathbb{R})}+\frac{c}{2}\left\|y_{1, x}-u_{x}\right\|_{H^{-1}(\mathbb{R})}\left\|y_{1, x}+u_{x}\right\|_{H^{s-1}(\mathbb{R})} \\
& +\frac{1}{2}\left\|y_{2}-\gamma\right\|_{L^{2}(\mathbb{R})}\left\|y_{2}+\gamma\right\|_{L^{\infty}(\mathbb{R})}+\frac{c}{2}\left\|y_{2, x}-\gamma_{x}\right\|_{H^{-1}(\mathbb{R})}\left\|y_{2, x}+\gamma_{x}\right\|_{H^{s-1}(\mathbb{R})} \\
& +c\left\|y_{1, x}\right\|_{H^{s-1}(\mathbb{R})}\left\|y_{2, x}-\gamma_{x}\right\|_{H^{-1}(\mathbb{R})}+c\left\|y_{1, x}-u_{x}\right\|_{H^{-1}(\mathbb{R})}\left\|\gamma_{x}\right\|_{H^{s-1}(\mathbb{R})} \\
& +\left\|y_{1, x}\right\|_{H^{s-1}(\mathbb{R})}\left\|y_{2}-\gamma\right\|_{H^{-1}(\mathbb{R})}+c\left\|y_{1, x}-u_{x}\right\|_{H^{-1}(\mathbb{R})}\|\gamma\|_{H^{s-1}(\mathbb{R})} \\
= & (3+4 c)\left(\|y\|_{H^{s}(\mathbb{R}) \times H^{s}(\mathbb{R})}+\|z\|_{H^{s}(\mathbb{R}) \times H^{s}(\mathbb{R})}\right)\|y-z\|_{L^{2}(\mathbb{R}) \times L^{2}(\mathbb{R}),}
\end{aligned}
$$

where we applied Lemma 2.2 with $r=s-1, t=-1$. This proves Lemma 2.4.

Lemma 2.5. Let $z_{0} \in H^{s}(\mathbb{R}) \times H^{s}(\mathbb{R}), s>2$ and let $T>0$ be the maximal existence time of the corresponding solution $z=\left(\begin{array}{l}u \\ \gamma\end{array}\right)$ to Eq. (2.1). Then (2.3) holds for every $t \in(0, T)$.

Proof. Applying Lemma 2.4 and a simple density argument, we only need to show that the above lemma holds for some $s>2$. Here we assume $s=3$ to prove the lemma. Differentiating Eq. (2.1) with respect to $x$ and using the identity $\partial_{x}^{2} p * f=p * f-f$, we have

$$
\left\{\begin{array}{l}
u_{t x}+u u_{x x}+u_{x}^{2}=\epsilon u_{x x x}+f-p * f, \\
\gamma_{t x}+u_{x} \gamma_{x}+u \gamma_{x x}=-\partial_{x} p *\left(\left(u_{x} \gamma_{x}\right)_{x}+u_{x} \gamma\right),
\end{array}\right.
$$

where $f=u^{2}+\frac{1}{2} u_{x}^{2}+\frac{1}{2} \gamma^{2}-\frac{1}{2} \gamma_{x}^{2}$. Denote $g=\left(u_{x} \gamma_{x}\right)_{x}+u_{x} \gamma$ and $E(t)=\int\left(u^{2}+u_{x}^{2}+\gamma^{2}+\gamma_{x}^{2}\right) d x$. Using Eq. (2.1) and Eq. (2.4), and integrating by parts, we obtain

$$
\begin{aligned}
\frac{d}{d t} E(t)= & 2 \int_{\mathbb{R}}\left(u u_{t}+u_{x} u_{x t}+\gamma \gamma_{t}+\gamma_{x} \gamma_{x t}\right) d x \\
= & 2 \int_{\mathbb{R}}\left[u\left(-u u_{x}-\partial_{x} p * f\right)+u_{x}\left(-u u_{x x}-u_{x}^{2}+f-p * f\right)\right. \\
& \left.+\gamma\left(-u \gamma_{x}-p * g\right)+\gamma_{x}\left(-u_{x} \gamma_{x}-u \gamma_{x x}-\partial_{x} p * g\right)\right] d x+2 \epsilon \int_{\mathbb{R}}\left(u u_{x x}+u_{x} u_{x x x}\right) d x \\
= & 2 \int_{\mathbb{R}}\left(-u \partial_{x} p * f-\frac{1}{2} u_{x}^{3}+u_{x} f-u_{x} p * f+\frac{1}{2} u_{x} \gamma^{2}\right.
\end{aligned}
$$




$$
\begin{aligned}
& \left.-\gamma p * g-\frac{1}{2} u_{x} \gamma_{x}^{2}-\gamma_{x} \partial_{x} p * g\right) d x-2 \epsilon \int_{\mathbb{R}}\left(u_{x}^{2}+u_{x x}^{2}\right)(s, x) d x \\
= & -2 \epsilon \int_{\mathbb{R}}\left(u_{x}^{2}+u_{x x}^{2}\right)(s, x) d x .
\end{aligned}
$$

Integrating the above equality over $(0, t)$, we get (2.3).

If $z \in H^{s}(\mathbb{R}) \times H^{s}(\mathbb{R}), s>2$, then by the above lemma we have the following useful inequality

$$
\begin{aligned}
\|u(t, \cdot)\|_{L^{\infty}(\mathbb{R})}^{2}+\|\gamma(t, \cdot)\|_{L^{\infty}(\mathbb{R})}^{2} & \leqslant \frac{1}{2}\|u\|_{H^{1}(\mathbb{R})}^{2}+\frac{1}{2}\|\gamma\|_{H^{1}(\mathbb{R})}^{2} \\
\leqslant \frac{1}{2}\left(\left\|u_{0}\right\|_{H^{1}(\mathbb{R})}^{2}+\left\|\gamma_{0}\right\|_{H^{1}(\mathbb{R})}^{2}\right) & =\frac{1}{2}\left\|z_{0}\right\|_{H^{1}(\mathbb{R}) \times H^{1}(\mathbb{R})}^{2}
\end{aligned}
$$

for all $t \in[0, T)$.

Now we present the blow-up scenario for the strong solutions to Eq. (2.1).

Lemma 2.6. Let $z_{0}=\left(\begin{array}{c}u_{0} \\ \gamma_{0}\end{array}\right) \in H^{s}(\mathbb{R}) \times H^{s}(\mathbb{R}), s>2$ be given and assume that $T$ is the maximal existence time of the corresponding solution $z=\left(\begin{array}{l}u \\ \gamma\end{array}\right)$ of Eq. (2.1) with the initial data $z_{0}$. Then the $H^{s}(\mathbb{R}) \times H^{s}(\mathbb{R})$-norm of $z(t, \cdot)$ blows up if and only if

$$
\limsup _{t \rightarrow T}\left\{\left\|u_{x}(t, \cdot)\right\|_{L^{\infty}(\mathbb{R})}+\left\|\gamma_{x}(t, \cdot)\right\|_{L^{\infty}(\mathbb{R})}\right\}=\infty .
$$

Proof. Let $z=\left(\begin{array}{l}u \\ \gamma\end{array}\right)$ be the solution to Eq. (2.1) with the initial data $z_{0} \in H^{s}(\mathbb{R}) \times H^{s}(\mathbb{R}), s>2$, and let $T$ be the maximal existence time of the corresponding solution $z$, which is guaranteed by Lemma 2.4. Throughout this proof, $c>0$ stands for a generic constant depending only on $s$.

Applying the operator $\Lambda^{s}$ to the first equation in Eq. (2.2), multiplying by $\Lambda^{s} u$, and integrating over $\mathbb{R}$, we obtain

$$
\frac{d}{d t}\|u\|_{H^{s}(\mathbb{R})}^{2}=-2\left(u u_{x}, u\right)_{s}-2(u, f(u, \gamma))_{s}+2 \epsilon\left(u_{x x}, u\right)_{s}
$$

where

$$
f(u, \gamma)=\partial_{x}\left(1-\partial_{x}^{2}\right)^{-1}\left(u^{2}+\frac{1}{2} u_{x}^{2}+\frac{1}{2} \gamma^{2}-\frac{1}{2} \gamma_{x}^{2}\right) .
$$

From the proof of Theorem 3.1 in [35], we know that

$$
\left|\left(u u_{x}, u\right)_{s}\right| \leqslant c\left\|u_{x}\right\|_{L^{\infty}(\mathbb{R})}\|u\|_{H^{s}(\mathbb{R})}^{2}
$$

and

$$
\left|(f(u, \gamma), u)_{s}\right| \leqslant c\left(\left\|u_{x}\right\|_{L^{\infty}(\mathbb{R})}+\left\|\gamma_{x}\right\|_{L^{\infty}(\mathbb{R})}+\left\|z_{0}\right\|_{H^{1}(\mathbb{R}) \times H^{1}(\mathbb{R})}\right)\left(\|\gamma\|_{H^{s}(\mathbb{R})}^{2}+\|u\|_{H^{s}(\mathbb{R})}^{2}\right) .
$$

Note that $2 \epsilon\left(u_{x x}, u\right)_{s}=-2 \epsilon\left\|u_{x}\right\|_{H^{s}(\mathbb{R})} \leqslant 0$. Combining the above three inequalities, we get

$$
\frac{d}{d t}\|u\|_{H^{s}(\mathbb{R})}^{2} \leqslant c\left(\left\|u_{x}\right\|_{L^{\infty}(\mathbb{R})}+\left\|\left.\gamma_{x}\right|_{L^{\infty}(\mathbb{R})}+\right\| z_{0} \|_{H^{1}(\mathbb{R}) \times H^{1}(\mathbb{R})}\right)\left(\|\gamma\|_{H^{s}(\mathbb{R})}^{2}+\|u\|_{H^{s}(\mathbb{R})}^{2}\right) .
$$

From the proof of Theorem 3.1 in [35] again, we have

$$
\frac{d}{d t}\|\gamma\|_{H^{s}(\mathbb{R})}^{2} \leqslant c\left(\left\|u_{x}\right\|_{L^{\infty}(\mathbb{R})}+\left\|\gamma_{x}\right\|_{L^{\infty}(\mathbb{R})}+\left\|z_{0}\right\|_{H^{1}(\mathbb{R}) \times H^{1}(\mathbb{R})}\right)\left(\|\gamma\|_{H^{s}(\mathbb{R})}^{2}+\|u\|_{H^{s}(\mathbb{R})}^{2}\right) .
$$

By (2.6) and (2.7), we obtain

$$
\frac{d}{d t}\left(\|u\|_{H^{s}(\mathbb{R})}^{2}+\|\gamma\|_{H^{s}(\mathbb{R})}^{2}\right) \leqslant c\left(\left\|u_{x}\right\|_{L^{\infty}(\mathbb{R})}+\left\|\gamma_{x}\right\|_{L^{\infty}(\mathbb{R})}+\left\|z_{0}\right\|_{H^{1}(\mathbb{R}) \times H^{1}(\mathbb{R})}\right)\left(\|\gamma\|_{H^{s}(\mathbb{R})}^{2}+\|u\|_{H^{s}(\mathbb{R})}^{2}\right) .
$$

If there exists $M>0$ such that $\limsup _{t \rightarrow T}\left\|u_{x}(t, \cdot)\right\|_{L^{\infty}}+\left\|\gamma_{x}(t, \cdot)\right\|_{L^{\infty}} \leqslant M$, then an application of Gronwall's inequality and the assumption of the theorem yield

$$
\|u\|_{H^{s}(\mathbb{R})}^{2}+\|\gamma\|_{H^{s}(\mathbb{R})}^{2} \leqslant \exp \left(c M_{1} t\right)\left(\left\|u_{0}\right\|_{H^{s}(\mathbb{R})}^{2}+\left\|\gamma_{0}\right\|_{H^{s}(\mathbb{R})}^{2}\right),
$$

where $M_{1}=M+\left\|z_{0}\right\|_{H^{1}(\mathbb{R}) \times H^{1}(\mathbb{R})}$. 
On the other hand, by Sobolev's imbedding theorem, we see that if

$$
\limsup _{t \rightarrow T}\left\{\left\|u_{x}(t, \cdot)\right\|_{L^{\infty}(\mathbb{R})}+\left\|\gamma_{x}(t, \cdot)\right\|_{L^{\infty}(\mathbb{R})}\right\}=\infty,
$$

then the solution will blow up in finite time. This completes the proof of the lemma.

Consider now the following initial value problem

$$
\begin{cases}q_{t}=u(t, q), & t \in[0, T), \\ q(0, x)=x, & x \in \mathbb{R}\end{cases}
$$

where $u$ denotes the first component of the solution $z$ to Eq. (1.1). The introduction of $q$ is suggested by working in the Lagrangian framework, cf. [1] and [21] (see also [45]). Applying classical results in the theory of ordinary differential equations, one can obtain the following result on $q$ which is crucial in the proof of blow-up scenarios.

Lemma 2.7. (See [11].) Let $u \in C\left([0, T) ; H^{s}(\mathbb{R})\right) \cap C^{1}\left([0, T) ; H^{s-1}(\mathbb{R})\right), s \geqslant 2$. Then Eq. (2.8) has a unique solution $q \in C^{1}([0, T) \times \mathbb{R} ; \mathbb{R})$. Moreover, the map $q(t, \cdot)$ is an increasing diffeomorphism of $\mathbb{R}$ with

$$
q_{x}(t, x)=\exp \left(\int_{0}^{t} u_{x}(s, q(s, x)) d s\right)>0, \quad \forall(t, x) \in[0, T) \times \mathbb{R} .
$$

Lemma 2.8. Let $z_{0} \in H^{s}(\mathbb{R}) \times H^{s}(\mathbb{R}), s>2$ and $T>0$ be the maximal existence time of the corresponding solution $z=\left(\begin{array}{l}u \\ \gamma\end{array}\right)$ to Eq. (2.1) guaranteed by Lemma 2.4. If $\rho_{0}=\gamma_{0}-\gamma_{0, x x}$ does not change sign on $\mathbb{R}$, then we have for all $t \in[0, T)$,

$$
\left\|\gamma_{x}(t, \cdot)\right\|_{L^{\infty}(\mathbb{R})} \leqslant\|\gamma(t, \cdot)\|_{L^{\infty}(\mathbb{R})} \leqslant \frac{\sqrt{2}}{2}\left\|z_{0}\right\|_{H^{1}(\mathbb{R}) \times H^{1}(\mathbb{R})} .
$$

Moreover, if $\rho_{0} \in L^{1}(\mathbb{R})$ we have

$$
\|\gamma(t, \cdot)\|_{L^{1}(\mathbb{R})}=\|\rho(t, \cdot)\|_{L^{1}(\mathbb{R})}=\left\|\rho_{0}\right\|_{L^{1}(\mathbb{R})}, \quad \forall t \in[0, T) .
$$

Proof. Note that $\rho=\gamma-\gamma_{x x}$. Then, form the second equation of (2.4) we have

$$
\rho_{t}+(u \rho)_{x}=0 .
$$

By (2.8) and (2.11), we get

$$
\frac{d}{d t}\left(\rho(t, q(t, x)) q_{x}(t, x)\right)=\left(\rho_{t}+\rho_{x} q_{t}\right) q_{x}+\rho q_{x t}=\left(\rho_{t}+\rho_{x} q_{t}+\rho u_{x}\right) q_{x}=0 .
$$

Thus, we obtain

$$
\rho(t, q(t, x)) q_{x}(t, x)=\rho_{0}(x) .
$$

In view of Lemma 2.7, if $\rho_{0}$ does not change sign on $\mathbb{R}$, then for any $t \in[0, T), \rho(t, x)$ and $\rho_{0}$ have the same sign by (2.12). Since $\gamma=p * \rho$ where $p=\frac{1}{2} e^{-|x|}$, we deduce

$$
\begin{aligned}
\left|\gamma_{x}\right| & =\frac{1}{2}\left|e^{x} \int_{x}^{\infty} e^{-y} \rho(y) d y-e^{-x} \int_{-\infty}^{x} e^{y} \rho(y) d y\right| \\
& \leqslant \frac{1}{2}\left|e^{x} \int_{x}^{\infty} e^{-y} \rho(y) d y+e^{-x} \int_{-\infty}^{x} e^{y} \rho(y) d y\right|=|\gamma| .
\end{aligned}
$$

Then, by (2.5) we obtain (2.9).

In view of (2.12) and Lemma 2.7, if $\rho_{0}$ does not change sign on $\mathbb{R}$ and $\rho_{0} \in L^{1}(\mathbb{R})$, by the relation $\gamma=p * \rho$ we have that $\gamma(t, \cdot)$ does not change sign on $\mathbb{R}$. Then, by Eq. (2.1) and (2.11) we obtain (2.10). This completes the proof of the lemma. 
From Lemmas 2.6 and 2.8, we obtain the following corollary.

Corollary 2.1. Let $z_{0}=\left(\begin{array}{c}u_{0} \\ \gamma_{0}\end{array}\right) \in H^{s}(\mathbb{R}) \times H^{s}(\mathbb{R}), s>2$ be given. Assume that $T$ is the maximal existence time of the corresponding solution $z=\left(\begin{array}{l}u \\ \gamma\end{array}\right)$ to Eq. (2.1) with the initial data $z_{0}$. If $\rho_{0}=\gamma_{0}-\gamma_{0, x x}$ does not change sign on $\mathbb{R}$, then the $H^{s} \times H^{s}$-norm of $z(t, \cdot)$ blows up on $[0, T)$ if and only if

$$
\limsup _{t \rightarrow T}\left\|u_{x}(t, \cdot)\right\|_{L^{\infty}(\mathbb{R})}=\infty
$$

Lemma 2.9. Let $z_{0} \in H^{s}(\mathbb{R}) \times H^{s}(\mathbb{R}), s>2$ and let $T>0$ be the maximal existence time of the corresponding solution $z=\left(\begin{array}{l}u \\ \gamma\end{array}\right)$ to Eq. (2.1) guaranteed by Lemma 2.4. If $\rho_{0}=\gamma_{0}-\gamma_{0, x x}$ does not change sign on $\mathbb{R}$, then we have

$$
\left\|u_{x}(t, \cdot)\right\|_{L^{\infty}(\mathbb{R})} \leqslant C\left(t+e^{C t}\right), \quad \forall t \in[0, T)
$$

where $C$ depends on $\epsilon$ and $\left\|z_{0}\right\|_{H^{2}(\mathbb{R}) \times H^{2}(\mathbb{R})}$, but does not depend on $t$.

Proof. By Lemma 2.5, for $t \in[0, T)$ we have that $\|z(t, \cdot)\|_{H^{1}(\mathbb{R}) \times H^{1}(\mathbb{R})} \leqslant C$. Differentiating the first equation in (2.4) with respect to $x$ and using the identity $\partial_{x}^{2} p * f=p * f-f$, we get

$$
u_{t x x}+u u_{x x x}-\epsilon u_{x x x x}=2 u u_{x}-2 u_{x} u_{x x}+\gamma \gamma_{x}-\gamma_{x} \gamma_{x x}-\partial_{x} p * f,
$$

where $f=u^{2}+\frac{1}{2} u_{x}^{2}+\frac{1}{2} \gamma^{2}-\frac{1}{2} \gamma_{x}^{2}$. By Lemma 2.5 and Hölder's inequality, we obtain

$$
\left\|\partial_{x} p * f\right\|_{L^{2}(\mathbb{R})}^{2} \leqslant\|f\|_{L^{1}(\mathbb{R})}^{2} \leqslant\left\|z_{0}\right\|_{H^{1}(\mathbb{R}) \times H^{1}(\mathbb{R})}^{4},
$$

where we have used $\left\|\partial_{x} p\right\|_{L^{2}(\mathbb{R})} \leqslant 1$. By (2.3), (2.5) and Lemma 2.8, in view of (2.14), we find

$$
\begin{aligned}
& \frac{1}{2} \frac{d}{d t} \int_{\mathbb{R}} u_{x x}^{2} d x+\epsilon \int_{\mathbb{R}} u_{x x x}^{2} d x \\
& =\int_{\mathbb{R}} u_{x x}\left(-u u_{x x x}+2 u u_{x}-2 u_{x} u_{x x}+\gamma \gamma_{x}-\gamma_{x} \gamma_{x x}-\partial_{x} p * f\right) d x \\
& =2 \int_{\mathbb{R}} u u_{x} u_{x x} d x+3 \int_{\mathbb{R}} u u_{x x} u_{x x x} d x+\int_{\mathbb{R}} \gamma \gamma_{x} u_{x x} d x+\frac{1}{2} \int_{\mathbb{R}}\left(\gamma_{x}\right)^{2} u_{x x x} d x-\int_{\mathbb{R}} u_{x x} \partial_{x} p * f d x \\
& \leqslant\|u\|_{L^{\infty}(\mathbb{R})}\left\|u_{x}\right\|_{H^{1}(\mathbb{R})}^{2}+\frac{\epsilon}{2}\left\|u_{x x x}\right\|_{L^{2}(\mathbb{R})}^{2}+\frac{9\|u\|_{L^{\infty}(\mathbb{R})}^{2}}{2 \epsilon}\left\|u_{x x}\right\|_{L^{2}(\mathbb{R})}^{2}+\frac{\|\gamma\|_{L^{\infty}(\mathbb{R})}}{2}\left(\left\|\gamma_{x}\right\|_{L^{2}(\mathbb{R})}^{2}+\left\|u_{x x}\right\|_{L^{2}(\mathbb{R})}^{2}\right) \\
& \quad+\frac{1}{2}\left(\left\|u_{x x}\right\|_{L^{2}(\mathbb{R})}^{2}+\left\|z_{0}\right\|_{H^{1}(\mathbb{R}) \times H^{1}(\mathbb{R})}^{4}\right)+\frac{\epsilon}{2}\left\|u_{x x x}\right\|_{L^{2}(\mathbb{R})}^{2}+\frac{1}{4 \epsilon}\left\|\gamma_{x}\right\|_{L^{4}(\mathbb{R})}^{4} \\
& \leqslant C_{1} \int_{\mathbb{R}} u_{x x}^{2} d x+C_{2}+\epsilon \int_{\mathbb{R}} u_{x x x}^{2} d x,
\end{aligned}
$$

here $C_{1}$ and $C_{2}$ only depend on $\epsilon$ and $\left\|z_{0}\right\|_{H^{1}(\mathbb{R}) \times H^{1}(\mathbb{R})}$. Then, by (2.15) and Gronwall's inequality, we have

$$
\left\|u_{x x}(t, \cdot)\right\|_{L^{2}(\mathbb{R})}^{2} \leqslant e^{C_{1} t}\left\|u_{0, x x}\right\|_{L^{2}(\mathbb{R})}^{2}+C_{2} t .
$$

Combining this with (2.3) shows that there exists a positive constant $C=C\left(\epsilon,\left\|z_{0}\right\|_{H^{2}(\mathbb{R}) \times H^{2}(\mathbb{R})}\right)$ such that

$$
\|u(t, \cdot)\|_{H^{2}(\mathbb{R})} \leqslant C\left(t+e^{C t}\right), \quad \forall t \in[0, T),
$$

which implies that $\left\|u_{x}(t, \cdot)\right\|_{L^{\infty}(\mathbb{R})} \leqslant C\left(t+e^{C t}\right)$ for all $t \in[0, T)$.

Combining Lemmas 2.3-2.9 and Corollary 2.1, using Kato's semigroup approach we have that Eq. (2.1) has a global strong solution and (2.3) holds true for all $t \geqslant 0$. This completes the proof of Theorem 2.1. 


\section{Uniform a priori estimates}

Let $z_{0} \in H^{1}(\mathbb{R}) \times H^{1}(\mathbb{R})$ and let $z_{\epsilon}(t, x)$ be the solution to $(2.1)$. The long-time existence of $z_{\epsilon}(t, x)$ is guaranteed by Theorem 2.1. Moreover, it satisfies the energy identity (2.3). To obtain the compactness of this approximate solution sequence $\left\{z_{\epsilon}(t, x)\right\}$ in $L_{l o c}^{2}\left(\mathbb{R}_{+} ; H_{l o c}^{1}(\mathbb{R}) \times H_{l o c}^{1}(\mathbb{R})\right)$, a priori estimates in addition to (2.3) are needed. In this section, we derive the uniform one-sided supernorm estimate and the space-time higher integrability estimate on $\partial_{x} u_{\epsilon}(t, x)$, which are essential for our compactness argument.

We denote $q_{\epsilon}=\frac{\partial u_{\epsilon}}{\partial x}, \pi_{\epsilon}=\frac{\partial \gamma_{\epsilon}}{\partial x}$ and $\rho_{\epsilon}=\gamma_{\epsilon}-\gamma_{\epsilon} x x$ in the following text.

Remark 3.1. Note that $\rho_{0, \epsilon}=\phi_{\epsilon} * \rho_{0} \geqslant 0$ and $\left\|\rho_{0, \epsilon}\right\|_{L^{1}(\mathbb{R})} \leqslant\left\|\rho_{0}\right\|_{\mathcal{M}(\mathbb{R})}$, for all $\epsilon>0$, provided $\rho_{0} \in \mathcal{M}^{+}(\mathbb{R})$. Thus, by Lemma 2.4 and Lemma 2.8 , we have

$$
\left\|\pi_{\epsilon}(t, \cdot)\right\|_{L^{\infty}(\mathbb{R})} \leqslant\left\|\gamma_{\epsilon}(t, \cdot)\right\|_{L^{\infty}(\mathbb{R})} \leqslant \frac{\sqrt{2}}{2}\left\|z_{0, \epsilon}\right\|_{H^{1}(\mathbb{R}) \times H^{1}(\mathbb{R})} \leqslant \frac{\sqrt{2}}{2}\left\|z_{0}\right\|_{H^{1}(\mathbb{R}) \times H^{1}(\mathbb{R})} .
$$

Using Remark 3.1, we have the following uniform one-side supernorm estimate on $q_{\epsilon}(t, x)$.

Lemma 3.1. If $\rho_{0} \in \mathcal{M}^{+}(\mathbb{R})$, then for each $t>0$ and $x \in \mathbb{R}$, we have

$$
q_{\epsilon}(t, x) \leqslant \frac{2}{t}+\sqrt{2}\left\|z_{0}\right\|_{H^{1}(\mathbb{R}) \times H^{1}(\mathbb{R})} .
$$

Proof. By the first equation of (2.4), we have

$$
\frac{\partial q_{\epsilon}}{\partial t}+u_{\epsilon} \frac{\partial q_{\epsilon}}{\partial x}+\frac{1}{2}\left(q_{\epsilon}\right)^{2}-\epsilon \partial_{x x} q_{\epsilon}=\left(u_{\epsilon}\right)^{2}+\frac{1}{2}\left(\gamma_{\epsilon}\right)^{2}-\frac{1}{2}\left(\pi_{\epsilon}\right)^{2}-p * f,
$$

where $f=\left(u_{\epsilon}\right)^{2}+\frac{1}{2}\left(q_{\epsilon}\right)^{2}+\frac{1}{2} \gamma_{\epsilon}^{2}-\frac{1}{2} \pi_{\epsilon}^{2}$. By Lemma 2.4, we get

$$
\left\|\left(u_{\epsilon}\right)^{2}(t, \cdot)\right\|_{L^{\infty}(\mathbb{R})}+\left\|\left(\gamma_{\epsilon}\right)^{2}(t, \cdot)\right\|_{L^{\infty}(\mathbb{R})} \leqslant \frac{1}{2}\left(\left\|u_{\epsilon}(t, \cdot)\right\|_{H^{1}(\mathbb{R})}^{2}+\left\|\gamma_{\epsilon}(t, \cdot)\right\|_{H^{1}(\mathbb{R})}^{2}\right) \leqslant \frac{1}{2}\left\|z_{0}\right\|_{H^{1}(\mathbb{R}) \times H^{1}(\mathbb{R})}^{2},
$$

and

$$
\begin{aligned}
\|(p * f)(t, \cdot)\|_{L^{\infty}(\mathbb{R})} & \leqslant\|p\|_{L^{\infty}(\mathbb{R})}\left\|\left(\left(u_{\epsilon}\right)^{2}+\frac{1}{2}\left(q_{\epsilon}\right)^{2}+\frac{1}{2} \gamma_{\epsilon}^{2}-\frac{1}{2} \pi_{\epsilon}^{2}\right)(t, \cdot)\right\|_{L^{1}(\mathbb{R})} \\
& \leqslant \frac{1}{2}\left\|z_{\epsilon}\right\|_{H^{1}(\mathbb{R}) \times H^{1}(\mathbb{R})}^{2} \leqslant \frac{1}{2}\left\|z_{0}\right\|_{H^{1}(\mathbb{R}) \times H^{1}(\mathbb{R})}^{2},
\end{aligned}
$$

where we have used $\|p\|_{L^{\infty}(\mathbb{R})}=\frac{1}{2}$. Due to the above two inequalities, we obtain

$$
\frac{\partial q_{\epsilon}}{\partial t}+u_{\epsilon} \frac{\partial q_{\epsilon}}{\partial x}+\frac{1}{2}\left(q_{\epsilon}\right)^{2}-\epsilon \partial_{x x} q_{\epsilon} \leqslant\left\|z_{0}\right\|_{H^{1}(\mathbb{R}) \times H^{1}(\mathbb{R})}^{2}, \quad \forall(t, x) \in \mathbb{R}_{+} \times \mathbb{R} .
$$

Now, let $F(t)=\frac{2}{t}+\sqrt{2}\left\|z_{0}\right\|_{H^{1}(\mathbb{R}) \times H^{1}(\mathbb{R})}$. It is obvious that for any $t>0$,

$$
\frac{d F}{d t}+\frac{1}{2} F^{2}=\left\|z_{0}\right\|_{H^{1}(\mathbb{R}) \times H^{1}(\mathbb{R})}^{2}+\frac{2 \sqrt{2}\left\|z_{0}\right\|_{H^{1}(\mathbb{R}) \times H^{1}(\mathbb{R})}}{t}>\left\|z_{0}\right\|_{H^{1}(\mathbb{R}) \times H^{1}(\mathbb{R})}^{2},
$$

$q_{\epsilon}(0, x) \leqslant\left\|z_{0 \epsilon}\right\|_{H^{2}(\mathbb{R}) \times H^{2}(\mathbb{R})}$, and $\lim _{t \rightarrow 0^{+}} F(t)=+\infty$. Then the comparison principle for parabolic equations yields

$$
q_{\epsilon}(t, x) \leqslant F(t), \quad \forall t>0, x \in \mathbb{R} .
$$

Therefore, the estimate (3.2) is proven.

Next, we derive the uniform local space-time higher integrability estimate for $q_{\epsilon}$. 
Lemma 3.2. Let $0<\alpha<1, T>0$ and $a, b \in \mathbb{R}, a<b$. Under the assumption of Theorem 1.1 , there exists a positive constant $C$ depending only on $\left\|z_{0}\right\|_{H^{1}(\mathbb{R}) \times H^{1}(\mathbb{R})}, \alpha, T$, $a$ and $b$, but being independent of $\epsilon$ such that

$$
\int_{0}^{T} \int_{a}^{b}\left|\frac{\partial u_{\epsilon}}{\partial x}(t, x)\right|^{2+\alpha} d x d t \leqslant C .
$$

Proof. Let $\chi \in C^{\infty}(\mathbb{R})$ be a cut-off function such that

$$
0 \leqslant \chi \leqslant 1, \quad \chi(x)= \begin{cases}1, & x \in[a, b], \\ 0, & x \in(-\infty, a-1] \cup[b+1, \infty) .\end{cases}
$$

Consider the map $\theta(\xi):=\xi(|\xi|+1)^{\alpha}, \xi \in \mathbb{R}$, which was introduced in [8]. Obviously,

$$
\begin{aligned}
& \theta^{\prime}(\xi)=((\alpha+1)|\xi|+1)(|\xi|+1)^{\alpha-1}, \\
& |\theta(\xi)| \leqslant|\xi|^{\alpha+1}+|\xi|, \quad 0<\theta^{\prime}(\xi) \leqslant(\alpha+1)|\xi|+1, \quad\left|\theta^{\prime \prime}(\xi)\right| \leqslant 2 \alpha, \\
& \xi \theta(\xi)-\frac{1}{2} \xi^{2} \theta^{\prime}(\xi) \geqslant \frac{1-\alpha}{2} \xi^{2}(|\xi|+1)^{\alpha} .
\end{aligned}
$$

Multiplying the first equation of (2.4) by $\chi \theta^{\prime}\left(q_{\epsilon}\right)$, using the chain rule, and integrating over $\prod_{T}:=[0, T] \times \mathbb{R}$, we get

$$
\begin{aligned}
\int_{\mathbb{R}} \chi & (x)\left(\theta\left(q_{\epsilon}(T, x)\right)-\theta\left(q_{\epsilon}(0, x)\right)\right) d x-\int_{\prod_{T}} q_{\epsilon} \chi(x) \theta\left(q_{\epsilon}\right) d x d t-\int_{\prod_{T}} u_{\epsilon} \chi^{\prime}(x) \theta\left(q_{\epsilon}\right) d x d t \\
& +\frac{1}{2} \int_{\prod_{T}} \chi(x) q_{\epsilon}^{2} \theta^{\prime}\left(q_{\epsilon}\right) d x d t+\epsilon \int_{\prod_{T}} \frac{\partial q_{\epsilon}}{\partial x} \chi^{\prime}(x) \theta^{\prime}\left(q_{\epsilon}\right) d x d t+\epsilon \int_{\prod_{T}}\left(\frac{\partial q_{\epsilon}}{\partial x}\right)^{2} \chi(x) \theta^{\prime \prime}\left(q_{\epsilon}\right) d x d t \\
= & \int_{\prod_{T}}\left(u_{\epsilon}^{2}+\frac{1}{2} \gamma_{\epsilon}^{2}-\frac{1}{2} \pi_{\epsilon}-P_{\epsilon}\right) \chi(x) \theta^{\prime}\left(q_{\epsilon}\right) d x d t,
\end{aligned}
$$

where $P_{\epsilon}=p *\left(u_{\epsilon}^{2}+\frac{1}{2} q_{\epsilon}^{2}+\frac{1}{2} \gamma_{\epsilon}^{2}-\frac{1}{2} \pi_{\epsilon}^{2}\right)$. Then

$$
\begin{aligned}
\int_{\prod_{T}} \chi q_{\epsilon} \theta\left(q_{\epsilon}\right) d x d t-\frac{1}{2} \int_{\prod_{T}} q_{\epsilon}^{2} \chi \theta^{\prime}\left(q_{\epsilon}\right) d x d t= & \int_{\mathbb{R}} \chi\left(\theta\left(q_{\epsilon}(T, x)\right)-\theta\left(q_{\epsilon}(0, x)\right)\right) d x-\int_{\prod_{T}} u_{\epsilon} \chi^{\prime}(x) \theta\left(q_{\epsilon}\right) d x d t \\
& +\epsilon \int_{\prod_{T}} \frac{\partial q_{\epsilon}}{\partial x} \chi^{\prime}(x) \theta^{\prime}\left(q_{\epsilon}\right) d x d t+\epsilon \int\left(\frac{\partial q_{\epsilon}}{\partial x}\right)^{2} \chi(x) \theta^{\prime \prime}\left(q_{\epsilon}\right) d x d t \\
& -\int_{\prod_{T}}\left(u_{\epsilon}^{2}+\frac{1}{2} \gamma_{\epsilon}^{2}-\frac{1}{2} \pi_{\epsilon}^{2}-P_{\epsilon}\right) \chi(x) \theta^{\prime}\left(q_{\epsilon}\right) d x d t
\end{aligned}
$$

By (3.6), we have

$$
\begin{aligned}
\int_{\prod_{T}} \chi q_{\epsilon} \theta\left(q_{\epsilon}\right) d x d t-\frac{1}{2} \int_{\prod_{T}} q_{\epsilon}^{2} \chi(x) \theta^{\prime}\left(q_{\epsilon}\right) d x d t & =\iint_{\prod_{T}} \chi(x)\left(q_{\epsilon} \theta\left(q_{\epsilon}\right)-\frac{1}{2} q_{\epsilon}^{2} \theta^{\prime}\left(q_{\epsilon}\right)\right) d x d t \\
& \geqslant \frac{1-\alpha}{2} \int_{\prod_{T}} \chi(x) q_{\epsilon}^{2}\left(\left|q_{\epsilon}\right|+1\right)^{\alpha} d x d t
\end{aligned}
$$

Using Hölder's inequality, the first part of (3.5) and Theorem 2.1, we obtain 


$$
\begin{gathered}
\int_{\mathbb{R}} \chi(x) \theta\left(q_{\epsilon}\right) d x \leqslant \int_{\mathbb{R}} \chi(x)\left(\left|q_{\epsilon}\right|^{\alpha+1}+\left|q_{\epsilon}\right|\right) d x \leqslant \\
\leqslant \chi\left\|_{L^{2 /(1-\alpha)}(\mathbb{R})}\right\| q_{\epsilon}(t, \cdot)\left\|_{L^{2}(\mathbb{R})}^{\alpha+1}+\right\| \chi\left\|_{L^{2}(\mathbb{R})}\right\| q_{\epsilon}(t, \cdot) \|_{L^{2}(\mathbb{R})} \\
\leqslant(b-a+2)^{(1-\alpha) / 2}\left\|z_{0}\right\|_{H^{1}(\mathbb{R}) \times H^{1}(\mathbb{R})}^{\alpha+1} \\
+(b-a+2)^{1 / 2}\left\|z_{0}\right\|_{H^{1}(\mathbb{R}) \times H^{1}(\mathbb{R})},
\end{gathered}
$$

and

$$
\begin{aligned}
\left|\int_{\prod_{T}} u_{\epsilon} \chi^{\prime}(x) \theta\left(q_{\epsilon}\right) d x d t\right| \leqslant & \left\|u_{\epsilon}\right\|_{L^{\infty}} \int_{\prod_{T}}\left|\chi^{\prime}(x)\right|\left(\left|q_{\epsilon}\right|^{\alpha+1}+\left|q_{\epsilon}\right|\right) d x d t \\
\leqslant & \frac{\sqrt{2}}{2}\left\|z_{0}\right\|_{H^{1}(\mathbb{R}) \times H^{1}(\mathbb{R})} \int_{0}^{T}\left\|\chi^{\prime}\right\|_{L^{2 /(1-\alpha)}(\mathbb{R})}\left\|q_{\epsilon}(t, \cdot)\right\|_{L^{2}(\mathbb{R})}^{\alpha+1} d t \\
& +\frac{\sqrt{2}}{2}\left\|z_{0}\right\|_{H^{1}(\mathbb{R}) \times H^{1}(\mathbb{R})} \int_{0}^{T}\left\|\chi^{\prime}\right\|_{L^{2}(\mathbb{R})}\left\|q_{\epsilon}(t, \cdot)\right\|_{L^{2}(\mathbb{R})} d t \\
\leqslant & \frac{\sqrt{2} T}{2}\left\|z_{0}\right\|_{H^{1}(\mathbb{R}) \times H^{1}(\mathbb{R})}^{\alpha+2}\left\|\chi^{\prime}\right\|_{L^{2 /(1-\alpha)(\mathbb{R})}}+\frac{\sqrt{2} T}{2}\left\|z_{0}\right\|_{H^{1}(\mathbb{R}) \times H^{1}(\mathbb{R})}^{2}\left\|\chi^{\prime}\right\|_{L^{2}(\mathbb{R})} .
\end{aligned}
$$

Moreover, we get

$$
\epsilon \int_{\prod_{T}} \frac{\partial q_{\epsilon}}{\partial x} \chi^{\prime}(x) \theta^{\prime}\left(q_{\epsilon}\right) d x d t=-\epsilon \int_{\prod_{T}} \chi^{\prime \prime}(x) \theta\left(q_{\epsilon}\right) d x d t .
$$

Again by Hölder's inequality, (2.5) and the first part of (3.5), we have

$$
\begin{aligned}
\left|\int_{\prod_{T}} \frac{\partial q_{\epsilon}}{\partial x} \chi^{\prime}(x) \theta^{\prime}\left(q_{\epsilon}\right) d x d t\right| & \leqslant \int_{\prod_{T}}\left|\chi^{\prime \prime}(x)\right|\left|\theta\left(q_{\epsilon}\right)\right| d x d t \\
& \leqslant \int_{\prod_{T}}\left(\left|q_{\epsilon}\right|^{\alpha+1}+\left|q_{\epsilon}\right|\right)\left|\chi^{\prime \prime}(x)\right| d x d t \\
& \leqslant \int_{0}^{T}\left(\left\|\chi^{\prime \prime}\right\|_{L^{2 /(1-\alpha)(\mathbb{R})}}\left\|q_{\epsilon}(t, \cdot)\right\|_{L^{2}(\mathbb{R})}^{\alpha+1}+\left\|\chi^{\prime \prime}\right\|_{L^{2}(\mathbb{R})}\left\|q_{\epsilon}(t, \cdot)\right\|_{L^{2}(\mathbb{R})}\right) d t \\
& \leqslant T\left(\left\|\chi^{\prime \prime}\right\|_{L^{2 /(1-\alpha)(\mathbb{R})}}\left\|z_{0}\right\|_{H^{1}(\mathbb{R}) \times H^{1}(\mathbb{R})}^{\alpha+1}+\left\|\chi^{\prime \prime}\right\|_{L^{2}(\mathbb{R})}\left\|z_{0}(t, \cdot)\right\|_{H^{1}(\mathbb{R}) \times H^{1}(\mathbb{R})}\right) .
\end{aligned}
$$

Using (2.3), (2.5) and the third part of (3.5), we deduce that

$$
\epsilon\left|\int_{\prod_{T}}\left(\frac{\partial q_{\epsilon}}{\partial x}\right)^{2} \chi(x) \theta^{\prime \prime}\left(q_{\epsilon}\right) d x d t\right| \leqslant 2 \alpha \epsilon\left|\int_{\prod_{T}}\left(\frac{\partial q_{\epsilon}}{\partial x}\right)^{2} d x d t\right| \leqslant \alpha\left\|z_{0}\right\|_{H^{1}(\mathbb{R}) \times H^{1}(\mathbb{R})}^{2} .
$$

Based on Lemma 3.2 and Remark 3.1, there exists a constant $L>0$ depending only on $\left\|z_{0}\right\|_{H^{1}(\mathbb{R}) \times H^{1}(\mathbb{R})}$, such that $\left\|\left(u_{\epsilon}\right)^{2}+\frac{1}{2}\left(\gamma_{\epsilon}\right)^{2}-\frac{1}{2} \pi_{\epsilon}^{2}-P_{\epsilon}\right\|_{L^{\infty}\left(\mathbb{R}_{+} \times \mathbb{R}\right)} \leqslant L$. Using the second part of (3.5), we obtain

$$
\begin{gathered}
\int_{\prod_{T}}\left(u_{\epsilon}^{2}+\frac{1}{2} \gamma_{\epsilon}^{2}-\frac{1}{2} \pi_{\epsilon}^{2}-P_{\epsilon}\right) \chi \theta^{\prime}\left(q_{\epsilon}\right) d x d t \\
\leqslant L \int_{\prod_{T}} \chi(x)\left((\alpha+1)\left|q_{\epsilon}\right|+1\right) d x d t
\end{gathered}
$$




$$
\begin{aligned}
& \leqslant L \int_{0}^{T}\left((\alpha+1)\|\chi\|_{L^{2}(\mathbb{R})}\left\|q_{\epsilon}(t, \cdot)\right\|_{L^{2}(\mathbb{R})}+\|\chi\|_{L^{1}(\mathbb{R})}\right) d t \\
& \leqslant L T\left((\alpha+1)(b-a+2)^{1 / 2}\left\|z_{0}\right\|_{H^{1}(\mathbb{R}) \times H^{1}(\mathbb{R})}+(b-a+2)\right) .
\end{aligned}
$$

From (3.7)-(3.13), we see that there exists a constant $c>0$ depending only on $\left\|z_{0}\right\|_{H^{1}(\mathbb{R}) \times H^{1}(\mathbb{R})}, \alpha, T>0, a$ and $b$, but being independent of $\epsilon$, such that

$$
\frac{1-\alpha}{2} \int_{\prod_{T}} \chi(x) q_{\epsilon}^{2}\left(\left|q_{\epsilon}\right|+1\right)^{\alpha} d x d t \leqslant c .
$$

Then

$$
\int_{0}^{T} \int_{a}^{b}\left|\frac{\partial u_{\epsilon}}{\partial x}(t, x)\right|^{2+\alpha} d x d t \leqslant \int_{\prod_{T}} \chi(x) q_{\epsilon}^{2}\left(\left|q_{\epsilon}\right|+1\right)^{\alpha} d x d t \leqslant \frac{2 c}{1-\alpha} .
$$

This completes the proof of the lemma.

\section{Precompactness and existence}

With the basic energy estimate and sign condition with $\rho_{\epsilon}$ in Section 2 and the uniform a priori estimates in Section 3, we are now ready to obtain the necessary compactness of the viscous approximate solutions $z_{\epsilon}(t, x)$. We start with the weak compactness in $L^{\infty}\left(\mathbb{R}_{+}, H^{1}(\mathbb{R}) \times H^{1}(\mathbb{R})\right)$.

In this section we denote $P_{1, \epsilon}=p *\left(u_{\epsilon}^{2}+\frac{1}{2} q_{\epsilon}^{2}+\frac{1}{2} \gamma_{\epsilon}^{2}-\frac{1}{2} \pi_{\epsilon}^{2}\right), P_{2, \epsilon}=p *\left(q_{\epsilon} \pi_{\epsilon}\right)$ and $P_{3, \epsilon}=p *\left(q_{\epsilon} \gamma_{\epsilon}\right)$, where $q_{\epsilon}=\partial_{x} u_{\epsilon}, \pi_{\epsilon}=\partial_{x} \gamma_{\epsilon}$ as in Section 3 .

Lemma 4.1. Under the assumption of Theorem 1.1, there exist a subsequence $\left\{z_{\epsilon_{k}}(t, x), P_{1, \epsilon_{k}}(t, x)\right\}$ of the sequence $\left\{z_{\epsilon}(t, x), P_{1, \epsilon}(t, x)\right\}$ and some functions $z(t, x), P_{1}(t, x)$ with $z=\left(\begin{array}{l}u \\ \gamma\end{array}\right) \in L^{\infty}\left(\mathbb{R}_{+}, H^{1}(\mathbb{R}) \times H^{1}(\mathbb{R})\right), P_{1} \in$ $L^{\infty}\left(\mathbb{R}_{+}, W^{1, \infty}(\mathbb{R})\right)$, such that

$$
z_{\epsilon_{k}} \rightarrow z \text { and } P_{1, \epsilon_{k}} \rightarrow P_{1}, \quad \text { as } k \rightarrow \infty,
$$

uniformly on any compact subset of $\mathbb{R}_{+} \times \mathbb{R}$.

Proof. It follows from Theorem 2.1 that $\left\{z_{\epsilon}(t, x)\right\}$ is uniformly bounded in $L^{\infty}\left(\mathbb{R}_{+}, H^{1}(\mathbb{R}) \times H^{1}(\mathbb{R})\right)$. Also, $\left\{\partial_{t} z_{\epsilon}(t, x)\right\}$ is uniformly bounded in $L^{2}([0, T] \times \mathbb{R}) \times L^{2}([0, T] \times \mathbb{R})$ for $T>0$. Indeed, by (2.3) and Eq. (2.1), we get

$$
\begin{aligned}
& \left\|P_{i, \epsilon}(t, \cdot)\right\|_{L^{2}(\mathbb{R})} \leqslant\|p\|_{L^{2}(\mathbb{R})}\left\|z_{\epsilon}(t, \cdot)\right\|_{H^{1}(\mathbb{R}) \times H^{1}(\mathbb{R})}^{2} \leqslant\left\|z_{0}\right\|_{H^{1}(\mathbb{R}) \times H^{1}(\mathbb{R})}^{2}, \\
& \left\|\partial_{x} P_{i, \epsilon}(t, \cdot)\right\|_{L^{2}(\mathbb{R})} \leqslant\left\|\partial_{x} p\right\|_{L^{2}(\mathbb{R})}\left\|z_{\epsilon}(t, \cdot)\right\|_{H^{1}(\mathbb{R}) \times H^{1}(\mathbb{R})}^{2} \leqslant\left\|z_{0}\right\|_{H^{1}(\mathbb{R}) \times H^{1}(\mathbb{R})}^{2},
\end{aligned}
$$

here $i=1,2,3$. Thus, by Corollary 8.4 in [42], there exist $z \in C\left((0, T) ; L^{\infty}(\mathbb{R}) \times L^{\infty}(\mathbb{R})\right)$ and a subsequence $\left\{z_{\epsilon_{k}}(t, x)\right\}$ such that $\left\{z_{\epsilon_{k}}(t, x)\right\}$ is weakly compact in $C\left((0, T) ; L^{\infty}(\mathbb{R}) \times L^{\infty}(\mathbb{R})\right)$ and $\left\{z_{\epsilon_{k}}(t, x)\right\}$ converges to $z(t, x)$ uniformly on each compact subset of $\mathbb{R}_{+} \times \mathbb{R}$ as $k \rightarrow \infty$. Moreover, $z(t, x) \in C((0, T) \times \mathbb{R}) \cap L^{\infty}\left((0, T) ; H^{1}(\mathbb{R}) \times\right.$ $\left.H^{1}(\mathbb{R})\right)$.

Next, we turn to the compactness of $\left\{P_{1, \epsilon}\right\}$. First, by (4.1) and (4.2), we have that $\left\{P_{1, \epsilon}\right\}$ is uniformly bounded in $L^{\infty}\left(\mathbb{R}_{+}, H^{1}(\mathbb{R})\right)$. Now we estimate $\partial_{t} P_{1, \epsilon}$. Note that

$$
\begin{aligned}
\frac{\partial P_{1 \epsilon}}{\partial t}= & p *\left(2 u_{\epsilon} \partial_{t} u_{\epsilon}+q_{\epsilon} \partial_{t} q_{\epsilon}+\gamma_{\epsilon} \partial_{t} \gamma_{\epsilon}-\pi_{\epsilon} \partial_{t} \pi_{\epsilon}\right) \\
= & p *\left(2 u_{\epsilon} \partial_{t} u_{\epsilon}+\gamma_{\epsilon} \partial_{t} \gamma_{\epsilon}\right)+p *\left(q_{\epsilon}\left(-u_{\epsilon} \partial_{x} q_{\epsilon}-\frac{1}{2} q_{\epsilon}^{2}+\epsilon \partial_{x}^{2} q_{\epsilon}+u_{\epsilon}^{2}+\frac{1}{2} \gamma_{\epsilon}^{2}-\frac{1}{2} \pi_{\epsilon}^{2}-P_{1 \epsilon}\right)\right) \\
& -p *\left(\pi_{\epsilon}\left(-u_{\epsilon} \partial_{x} \pi_{\epsilon}-P_{2 \epsilon}-\partial_{x} P_{3 \epsilon}\right)\right)=I_{1}+I_{2}-I_{3} .
\end{aligned}
$$


By (2.3), (2.5) and Eq. (2.1), we obtain that $I_{1}=p *\left(2 u_{\epsilon} \partial_{t} u_{\epsilon}+\gamma_{\epsilon} \partial_{t} \gamma_{\epsilon}\right)$ is uniformly bounded in $L^{2}([0, T] \times \mathbb{R})$ for any $T>0$. Since

$$
-u_{\epsilon} q_{\epsilon} \partial_{x} q_{\epsilon}-\frac{1}{2}\left(q_{\epsilon}\right)^{3}=-\frac{1}{2}\left(u_{\epsilon} q_{\epsilon}^{2}\right)_{x}
$$

and $q_{\epsilon} \partial_{x}^{2} q_{\epsilon}=\partial_{x}\left(q_{\epsilon} \partial_{x} q_{\epsilon}\right)-\left(\partial_{x} q_{\epsilon}\right)^{2}$, it follows that

$$
\begin{aligned}
I_{2} & =p *\left(q_{\epsilon}\left(-u_{\epsilon} \partial_{x} q_{\epsilon}-\frac{1}{2} q_{\epsilon}^{2}+\epsilon \partial_{x}^{2} q_{\epsilon}+u_{\epsilon}^{2}+\frac{1}{2} \gamma_{\epsilon}^{2}-\frac{1}{2} \pi_{\epsilon}^{2}-P_{1 \epsilon}\right)\right) \\
& =p *\left(q_{\epsilon}\left(u_{\epsilon}^{2}+\frac{1}{2} \gamma_{\epsilon}^{2}-\frac{1}{2} \pi_{\epsilon}^{2}-P_{1 \epsilon}\right)-\epsilon\left(\partial_{x} q_{\epsilon}\right)^{2}\right)-p *\left(\frac{1}{2} u_{\epsilon} q_{\epsilon}^{2}-\epsilon q_{\epsilon} \partial_{x} q_{\epsilon}\right)_{x} .
\end{aligned}
$$

By (2.3), (2.5) and (3.1), we get

$$
\begin{aligned}
\left\|I_{2}\right\|_{L^{2}((0, T) \times \mathbb{R})}^{2} \leqslant & \int_{0}^{T}\|p\|_{L^{2}(\mathbb{R})}^{2}\left\|\left(q_{\epsilon}\left(u_{\epsilon}^{2}+\frac{1}{2} \gamma_{\epsilon}^{2}-\frac{1}{2} \pi_{\epsilon}^{2}-P_{1 \epsilon}\right)-\epsilon\left(\partial_{x} q_{\epsilon}\right)^{2}\right)(t, \cdot)\right\|_{L^{1}(\mathbb{R})}^{2} d t \\
& +\int_{0}^{T}\left\|\partial_{x} p\right\|_{L^{2}(\mathbb{R})}^{2}\left\|\left(\frac{1}{2} u_{\epsilon} q_{\epsilon}^{2}-\epsilon q_{\epsilon} \partial_{x} q_{\epsilon}\right)(t, \cdot)\right\|_{L^{1}(\mathbb{R})}^{2} d t \\
\leqslant & C\left(T,\left\|z_{0}\right\|_{H^{1}(\mathbb{R}) \times H^{1}(\mathbb{R})}\right) .
\end{aligned}
$$

Next we estimate the last term. By (2.3), (2.5) and (3.1), in view of $u_{\epsilon} \pi_{\epsilon} \partial_{x} \pi_{\epsilon}=\frac{1}{2} \partial_{x}\left(u_{\epsilon} \pi_{\epsilon}^{2}\right)-\frac{1}{2} q_{\epsilon} \pi_{\epsilon}^{2}$, we have

$$
\begin{aligned}
\left\|I_{3}\right\|_{L^{2}((0, T) \times \mathbb{R})}^{2} \leqslant & \int_{0}^{T}\|p\|_{L^{2}(\mathbb{R})}^{2}\left\|\left(\pi_{\epsilon}\left(-P_{2 \epsilon}-\partial_{x} P_{3 \epsilon}\right)+\frac{1}{2} q_{\epsilon} \pi_{\epsilon}^{2}\right)(t, \cdot)\right\|_{L^{1}(\mathbb{R})}^{2} d t \\
& +\int_{0}^{T}\left\|\partial_{x} p\right\|_{L^{2}(\mathbb{R})}^{2}\left\|\left(-\frac{1}{2} u_{\epsilon} \pi_{\epsilon}^{2}\right)(t, \cdot)\right\|_{L^{1}(\mathbb{R})}^{2} d t \\
\leqslant & C\left(T,\left\|z_{0}\right\|_{H^{1}(\mathbb{R}) \times H^{1}(\mathbb{R})}\right) .
\end{aligned}
$$

Thus we prove that $\frac{\partial P_{1, \epsilon}}{\partial t}$ are uniformly bounded in $L^{2}((0, T) \times \mathbb{R})$ for every $T>0$. Consequently, by Corollary 8.4 in [42], there exist $P_{1} \in C\left((0, T) ; L^{\infty}(\mathbb{R}) \times L^{\infty}(\mathbb{R})\right)$ and a subsequence $\left\{P_{1, \epsilon_{k}}(t, x)\right\}$ such that $\left\{P_{1, \epsilon_{k}}(t, x)\right\}$ is weakly compact in $C\left((0, T) ; L^{\infty}(\mathbb{R}) \times L^{\infty}(\mathbb{R})\right)$ and $\left\{P_{1, \epsilon_{k}}(t, x)\right\}$ converges to $P_{1}(t, x)$ uniformly on each compact subset of $\mathbb{R}_{+} \times \mathbb{R}$ as $k \rightarrow \infty$. Moreover, $P_{1}(t, x) \in C((0, T) \times \mathbb{R}) \cap L^{\infty}\left((0, T) ; H^{1}(\mathbb{R}) \times H^{1}(\mathbb{R})\right)$.

By Hölder's inequality, we have

$$
\left\|P_{1 \epsilon}(t, \cdot)\right\|_{L^{\infty}(\mathbb{R})} \leqslant\|p\|_{L^{\infty}(\mathbb{R})}\left\|\left(u_{\epsilon}^{2}+\frac{1}{2} q_{\epsilon}^{2}+\frac{1}{2} \gamma_{\epsilon}^{2}-\frac{1}{2} \pi_{\epsilon}^{2}\right)(t, \cdot)\right\|_{L^{1}(\mathbb{R})} \leqslant \frac{1}{2}\left\|z_{0}\right\|_{H^{1}(\mathbb{R}) \times H^{1}(\mathbb{R})}^{2}
$$

and

$$
\left\|\partial_{x} P_{1 \epsilon}(t, \cdot)\right\|_{L^{\infty}(\mathbb{R})} \leqslant\left\|\partial_{x} p\right\|_{L^{\infty}(\mathbb{R})}\left\|\left(u_{\epsilon}^{2}+\frac{1}{2} q_{\epsilon}^{2}+\frac{1}{2} \gamma_{\epsilon}^{2}-\frac{1}{2} \pi_{\epsilon}^{2}\right)(t, \cdot)\right\|_{L^{1}(\mathbb{R})} \leqslant \frac{1}{2}\left\|z_{0}\right\|_{H^{1}(\mathbb{R}) \times H^{1}(\mathbb{R})}^{2} .
$$

Thus, we have $P_{1} \in L^{\infty}\left(\mathbb{R}_{+}, W^{1, \infty}(\mathbb{R})\right)$. This completes the proof of the lemma.

Next, we prove the stronger convergence result, i.e.

$$
\partial_{x} z_{\epsilon} \rightarrow \partial_{x} z \quad \text { as } \epsilon \rightarrow 0^{+} \text {in } L_{\text {loc }}^{2}\left(\mathbb{R}_{+} \times \mathbb{R}\right) \times L_{\text {loc }}^{2}\left(\mathbb{R}_{+} \times \mathbb{R}\right) .
$$


Lemma 4.2. Assume $z_{0}=\left(\begin{array}{c}u_{0} \\ \gamma_{0}\end{array}\right) \in H^{1}(\mathbb{R}) \times H^{1}(\mathbb{R})$ with $\rho_{0}=\gamma_{0}-\gamma_{0, x x} \in \mathcal{M}^{+}(\mathbb{R})$. Let $z_{\epsilon}=\left(\begin{array}{l}u_{\epsilon} \\ \gamma_{\epsilon}\end{array}\right)$ be the solution to Eq. (2.1) with initial data $z_{\epsilon, 0}=\left(\begin{array}{c}\left(\phi_{\epsilon} * u_{0}\right)(x) \\ \left(\phi_{\epsilon} * \gamma_{0}\right)(x)\end{array}\right)$ which is guaranteed by Theorem 2.1. Then, there exists a subsequence $\left\{\pi_{\epsilon_{k}}\right\}$ such that

$$
\pi_{\epsilon_{k}}(t, x) \rightarrow \pi(t, x)=\gamma_{x}(t, x) \text { as } k \rightarrow \infty \text { a.e. on } \mathbb{R}_{+} \times \mathbb{R} \text {. }
$$

Proof. Note that $\rho_{0}=\gamma_{0}-\gamma_{0, x x} \in \mathcal{M}^{+}(\mathbb{R})$. Then we have

$$
\rho_{0, \epsilon}:=\gamma_{0, \epsilon}-\gamma_{0, \epsilon, x x}=\phi_{\epsilon} * \rho_{0} \geqslant 0
$$

and

$$
\left\|\rho_{0, \epsilon}\right\|_{L^{1}(\mathbb{R})} \leqslant\left\|\rho_{0}\right\|_{\mathcal{M}}, \quad 0<\epsilon<1 .
$$

By Lemma 4.1, for fixed $T>0$, we have

$$
\gamma_{\epsilon_{k}} \rightarrow \gamma, \quad \text { in } H^{1}([0, T] \times \mathbb{R}), \quad \text { and } \quad \gamma_{\epsilon_{k}} \rightarrow \gamma, \quad \text { uniformly in } A \subset \mathbb{R}_{+} \times \mathbb{R},
$$

where $A$ is an any compact subset of $\mathbb{R}_{+} \times \mathbb{R}$. By Eq. (2.1), Lemma 2.8, (2.9)-(2.10) and (3.1), for fixed $t \in(0, T)$, we deduce that the sequence $\pi_{\epsilon}(t, \cdot) \in B V(\mathbb{R})$ with

$$
\mathbb{V}\left[\pi_{\epsilon}(t, \cdot)\right]=\left\|\gamma_{\epsilon, x x}(t, \cdot)\right\|_{L^{1}(\mathbb{R})} \leqslant\left\|\gamma_{\epsilon}(t, \cdot)\right\|_{L^{1}(\mathbb{R})}+\left\|\rho_{\epsilon}(t, \cdot)\right\|_{L^{1}(\mathbb{R})} \leqslant 2\left\|\rho_{0}\right\|_{\mathcal{M}(\mathbb{R})}
$$

and

$$
\left\|\pi_{\epsilon}(t, \cdot)\right\|_{L^{\infty}(\mathbb{R})} \leqslant\left\|\gamma_{\epsilon}(t, \cdot)\right\|_{L^{\infty}(\mathbb{R})} \leqslant \frac{\sqrt{2}}{2}\left\|z_{0, \epsilon}\right\|_{H^{1}(\mathbb{R}) \times H^{1}(\mathbb{R})} \leqslant \frac{\sqrt{2}}{2}\left\|z_{0}\right\|_{H^{1}(\mathbb{R}) \times H^{1}(\mathbb{R})} .
$$

Here $B V(\mathbb{R})$ is the space of functions with bounded variation and $\mathbb{V}(f)$ is the total variation of $f \in B V(\mathbb{R})$, cf. [41]. By Helly's theorem (see [41, p. 222]), there exists a subsequence, denoted again $\left\{\pi_{\epsilon_{k}}(t, \cdot)\right\}$, which converges at every point to some function $v(t, \cdot)$ of finite variation with $\mathbb{V}(v(t, \cdot)) \leqslant 2\left\|\rho_{0}\right\|_{\mathcal{M}(\mathbb{R})}$. Since for almost all $t \in(0, T), \pi_{\epsilon_{k}}(t, \cdot) \rightarrow \gamma_{x}(t, \cdot)$ in $\mathcal{D}^{\prime}(\mathbb{R})$, this enables us to identify $v(t, \cdot)$ with $\gamma_{x}(t, \cdot)$ for a.e. $t \in(0, T)$. Therefore $\pi_{\epsilon}(t, x) \rightarrow \pi(t, x)=\gamma_{x}(t, x)$ a.e. on $\mathbb{R}_{+} \times \mathbb{R}$, and $\mathbb{V}\left(\gamma_{x}(t, \cdot)\right)=\left\|\gamma_{x x}(t, \cdot)\right\|_{\mathcal{M}(\mathbb{R})} \leqslant 2\left\|\rho_{0}\right\|_{\mathcal{M}(\mathbb{R})}$, for a.e. $t \in(0, T)$. This completes the proof of the lemma.

Remark 4.1. From Lemma 3.2 and Lemma 4.1, we can deduce that there exist two functions $q \in L_{\text {loc }}^{p}\left(\mathbb{R}_{+} \times \mathbb{R}\right)$, $\overline{q^{2}} \in L_{l o c}^{r}\left(\mathbb{R}_{+} \times \mathbb{R}\right)$ such that

$$
q_{\epsilon_{k}} \rightarrow q \text { in } L_{\text {loc }}^{p}\left(\mathbb{R}_{+} \times \mathbb{R}\right), \quad q_{\epsilon_{k}}^{2} \rightarrow \overline{q^{2}} \text { in } L_{\text {loc }}^{r}\left(\mathbb{R}_{+} \times \mathbb{R}\right),
$$

for every $1<p<3,1<r<\frac{3}{2}$. Moreover,

$$
q^{2}(t, x) \leqslant \overline{q^{2}}(t, x), \quad \text { a.e. }(t, x) \in \mathbb{R}_{+} \times \mathbb{R} .
$$

In view of (4.4), we conclude that for any convex $\eta \in C^{1}(\mathbb{R})$ with $\eta^{\prime}$ bounded, Lipschitz continuous on $\mathbb{R}, \eta(0)=0$ and any $1<p<3$, we have

$$
\eta\left(q_{\epsilon_{k}}\right) \rightarrow \overline{\eta(q)} \text { in } L_{l o c}^{p}\left(\mathbb{R}_{+} \times \mathbb{R}\right) .
$$

By Lemma 2.8 and Lemmas 4.1-4.2, in view of (2.3), (2.5) and (4.4), we have

$$
\left(u_{\epsilon_{k}}^{2}+\frac{1}{2} \gamma_{\epsilon_{k}}^{2}-\frac{1}{2} \pi_{\epsilon_{k}}^{2}-P_{1, \epsilon_{k}}\right) \eta\left(q_{\epsilon}\right) \rightarrow\left(h\left(u, \gamma, \gamma_{x}\right)-P_{1}\right) \overline{\eta(q)} \quad \text { in } L_{l o c}^{p}\left(\mathbb{R}_{+} \times \mathbb{R}\right),
$$

where $h\left(u, \gamma, \gamma_{x}\right)=u^{2}+\frac{1}{2} \gamma^{2}-\frac{1}{2} \gamma_{x}^{2}$.

Multiplying Eq. (3.3) by $\eta^{\prime}\left(q_{\epsilon}\right)$, we get

$$
\begin{aligned}
\frac{\partial}{\partial t} \eta\left(q_{\epsilon}\right)+\frac{\partial}{\partial x}\left(u_{\epsilon} \eta\left(q_{\epsilon}\right)\right)= & q_{\epsilon} \eta\left(q_{\epsilon}\right)-\frac{1}{2} q_{\epsilon}^{2} \eta^{\prime}\left(q_{\epsilon}\right) \\
& +\left(u_{\epsilon}^{2}+\frac{1}{2} \gamma_{\epsilon}^{2}-\frac{1}{2} \pi_{\epsilon}^{2}-P_{1, \epsilon}\right) \eta^{\prime}\left(q_{\epsilon}\right)-\epsilon \partial_{x}\left(\eta^{\prime}\left(q_{\epsilon}\right) \partial_{x} q_{\epsilon}\right)-\epsilon \eta^{\prime \prime}\left(q_{\epsilon}\right)\left(\partial_{x} q_{\epsilon}\right)^{2} .
\end{aligned}
$$


Note that $\left\{\sqrt{\epsilon} \partial_{x} q_{\epsilon}\right\}$ is uniformly bounded in $L^{2}\left(\mathbb{R}_{+} \times \mathbb{R}\right)$ due to Theorem 2.1 . Taking $\epsilon \rightarrow 0$, due to the convexity of $\eta$, by Lemma 4.1 and (4.6)-(4.7) we obtain that

$$
\frac{\partial \overline{\eta(q)}}{\partial t}+\frac{\partial}{\partial x}(u \overline{\eta(q)}) \leqslant \overline{q \eta(q)}-\frac{1}{2} \overline{q^{2} \eta^{\prime}(q)}+\left(h\left(u, \gamma, \gamma_{x}\right)-P_{1}\right) \overline{\eta^{\prime}(q)}
$$

in the sense of distributions on $\mathbb{R}_{+} \times \mathbb{R}$, here $\bar{f}$ is the limit of $f_{\epsilon_{k}}$ in the sense of distributions on $\mathbb{R}_{+} \times \mathbb{R}$.

Using (3.3) and Lemmas 4.1-4.2, and letting $\epsilon \rightarrow 0$, we get

$$
\frac{\partial q}{\partial t}+\frac{\partial}{\partial x}(u q)=\frac{1}{2} \overline{q^{2}}+h\left(u, \gamma, \gamma_{x}\right)-P_{1},
$$

in the sense of distributions on $\mathbb{R}_{+} \times \mathbb{R}$.

The next lemma contains a renormalized formulation of (4.9).

Lemma 4.3. For any $\eta \in C^{1}(\mathbb{R})$ with $\eta^{\prime}$ bounded, Lipschitz continuous on $\mathbb{R}$ and $\eta(0)=0$, we have

$$
\frac{\partial \eta(q)}{\partial t}+\frac{\partial}{\partial x}(u \eta(q))=q \eta(q)+\left(\frac{1}{2} \overline{q^{2}}-q^{2}\right) \eta^{\prime}(q)+\left(h\left(u, \gamma, \gamma_{x}\right)-P_{1}\right) \eta^{\prime}(q),
$$

in the sense of distributions on $\left(\mathbb{R}_{+} \times \mathbb{R}\right)$, where $h\left(u, \gamma, \gamma_{x}\right)=u^{2}+\frac{1}{2} \gamma^{2}-\frac{1}{2} \gamma_{x}^{2}$.

Proof. Denote $q^{\epsilon}(t, x):=\left(q(t, \cdot) * \phi_{\epsilon}\right)(x)$. According to Lemma II.1 of [29], it follows from (4.9) that $q^{\epsilon}$ solves

$$
\frac{\partial q^{\epsilon}}{\partial t}+u \frac{\partial q^{\epsilon}}{\partial x}=\left(-q^{2}+\frac{1}{2} \overline{q^{2}}\right)+\left(h\left(u, \gamma, \gamma_{x}\right)-\bar{P}\right) * \phi_{\epsilon}+\tau_{\epsilon},
$$

where the error $\tau_{\epsilon}$ tends to zero in $L_{l o c}^{1}\left(\mathbb{R}_{+} \times \mathbb{R}\right)$. Multiplying (4.10) by $\eta^{\prime}\left(q^{\epsilon}\right)$, we get

$$
\frac{\partial \eta\left(q^{\epsilon}\right)}{\partial t}+\frac{\partial}{\partial x}\left(u \eta\left(q^{\epsilon}\right)\right)=\left(\left(\frac{1}{2} \overline{q^{2}}-q^{2}\right) * \phi_{\epsilon}\right) \eta^{\prime}\left(q^{\epsilon}\right)+q \eta\left(q^{\epsilon}\right)+\left(\left(h\left(u, \gamma, \gamma_{x}\right)-\bar{P}\right) * \phi_{\epsilon}+\tau_{\epsilon}\right) \eta^{\prime}\left(q^{\epsilon}\right) .
$$

Using the boundedness of $\eta, \eta^{\prime}$, we can send $\epsilon \rightarrow 0$ in (4.12) to obtain (4.11).

For the strong convergence of $\partial_{x} u_{\epsilon}$ we recall the following results.

Lemma 4.4. (See Appendix $C$ of [48].) Let $X$ be a separable reflexive Banach space and let $f^{n}$ be bounded in $L^{\infty}(0, T ; X)$ for some $T \in(0, \infty)$. We assume that $f^{n} \in C([0, T] ; Y)$ where $Y$ is a Banach space such that $X \hookrightarrow Y$, $Y^{\prime}$ is separable and dense in $X^{\prime}$. Furthermore, $\left(\phi, f^{n}(t)\right)_{Y^{\prime} \times Y}$ is uniformly continuous in $t \in[0, T]$ and uniformly in $n \geqslant 1$. Then $f^{n}$ is relatively compact in $C^{w}([0, T] ; X)$, the space of continuous functions from $[0, T]$ with values in $X$ when the latter space is equipped with its weak topology.

Remark 4.2. If the conditions which $f^{n}$ satisfies in Lemma 4.4 are replaced by the following conditions:

$$
f^{n} \in L^{\infty}(0, T ; X), \quad \partial_{t} f^{n} \in L^{p}(0, T ; Y) \quad \text { for some } p \in(1, \infty),
$$

and

$$
\left\|f^{n}\right\|_{L^{\infty}(0, T ; X)},\left\|\partial_{t} f^{n}\right\|_{L^{p}(0, T ; Y)} \leqslant C, \quad \forall n \geqslant 1,
$$

then the conclusion of Lemma 4.4 holds true.

Lemma 4.5. There holds

$$
\lim _{t \rightarrow 0^{+}} \int_{\mathbb{R}} q^{2}(t, x) d x=\lim _{t \rightarrow 0^{+}} \int_{\mathbb{R}} \overline{q^{2}}(t, x)=\int_{\mathbb{R}} u_{0, x}^{2}(x) d x .
$$


Proof. By Lemma 4.1 and Theorem 2.1, for any $T>0$, we have $u_{\epsilon} \in L^{\infty}\left((0, T) ; H^{1}(\mathbb{R})\right), u_{\epsilon, t}$ uniformly bounded in $L^{\infty}\left((0, T) ; L^{2}(\mathbb{R})\right)$ and $u_{\epsilon} \in C\left([0, T] ; H^{1}(\mathbb{R})\right)$. Then in view of Lemma 4.4, Remark 4.2 and the proof of Lemma 4.1, we have that $\left\{u_{\epsilon}\right\}$ contains a subsequence, we denote again by $\left\{u_{\epsilon_{k}}\right\}$, which converges weakly in $H^{1}(\mathbb{R})$ uniformly in $t$. The limit function is $u$. This implies that $u$ is weakly continuous from $(0, T)$ into $H^{1}(\mathbb{R})$, i.e.

$$
u \in C^{w}\left([0, T] ; H^{1}(\mathbb{R})\right) .
$$

Thus, we get

$$
q(t, \cdot) \rightarrow u_{0, x} \quad \text { in } L^{2}(\mathbb{R}) \text { as } t \rightarrow 0^{+} .
$$

Therefore

$$
\liminf _{t \rightarrow 0^{+}} \int_{\mathbb{R}} q^{2}(t, x) d x \geqslant \int_{\mathbb{R}} u_{0, x}^{2} d x .
$$

Similarly,

$$
\liminf _{t \rightarrow 0^{+}} \int_{\mathbb{R}} \gamma_{x}^{2}(t, x) d x \geqslant \int_{\mathbb{R}} \gamma_{0, x}^{2} d x .
$$

Then we have

$$
\liminf _{t \rightarrow 0^{+}} \int_{\mathbb{R}}\left(q^{2}(t, x)+\gamma_{x}^{2}(t, x)\right) d x \geqslant \int_{\mathbb{R}} u_{0, x}^{2}+\gamma_{0, x}^{2} d x .
$$

On the other hand, from (2.3) we obtain

$$
\begin{aligned}
& \int_{\mathbb{R}}\left(u^{2}(t, x)+\overline{q^{2}}(t, x)+\gamma^{2}(t, x)+\gamma_{x}^{2}(t, x)\right) d x \\
& \leqslant \liminf _{k \rightarrow \infty} \int_{\mathbb{R}}\left(\left(u_{\epsilon_{k}}\right)^{2}(t, x)+\left(u_{\epsilon_{k}, x}\right)^{2}(t, x)+\left(\gamma_{\epsilon_{k}}\right)^{2}(t, x)+\left(\gamma_{\epsilon_{k}, x}\right)^{2}(t, x)\right) d x \\
& \quad \leqslant \liminf _{k \rightarrow \infty} \int_{\mathbb{R}}\left(\left(u_{0}^{n_{k}}\right)^{2}(x)+\left(u_{0, x}^{n_{k}}\right)^{2}(x)+\left(\gamma_{0, \epsilon_{k}}\right)^{2}(x)+\left(\gamma_{0, \epsilon_{k}, x}\right)^{2}(x)\right) d x \\
& \quad=\int_{\mathbb{R}}\left(u_{0}^{2}+u_{0, x}^{2}+\gamma_{0}^{2}+\gamma_{0, x}^{2}\right) d x .
\end{aligned}
$$

Again using the continuity of $u, \gamma$ and by Lemma 4.1, we have

$$
\lim _{t \rightarrow 0^{+}} \int_{\mathbb{R}}\left(u^{2}(t, x)+\gamma^{2}(t, x)\right) d x=\int_{\mathbb{R}}\left(u_{0}^{2}+\gamma_{0}^{2}\right) d x .
$$

Hence

$$
\limsup _{t \rightarrow 0^{+}} \int_{\mathbb{R}}\left(\overline{u_{x}^{2}}(t, x)+\gamma^{2}(t, x)\right) d x \leqslant \int_{\mathbb{R}}\left(u_{0, x}^{2}+\gamma_{0, x}^{2}\right) d x .
$$

Clearly, by (4.5), (4.14)-(4.15) and Lemma 4.2, we get (4.13). This completes the proof of the lemma.

By (4.8), (4.10), Lemma 3.1, Lemmas 4.1-4.2 and Lemma 4.5, in view of $h\left(u, \gamma, \gamma_{x}\right) \in L^{\infty}\left(\mathbb{R}_{+} \times \mathbb{R}\right)$, we can obtain the following result.

Lemma 4.6. Under the assumption of Theorem 1.1, there holds

$$
q_{\epsilon}(t, x) \rightarrow q(t, x)=u_{x}(t, x) \text { a.e. on } \mathbb{R}_{+} \times \mathbb{R} \text {. }
$$


The proof of Lemma 4.6 goes along the lines of the analogous results in [51] or [8], so, we omit here.

Proof of Theorem 1.1. By the above results we have

$$
\begin{aligned}
& p *\left(u_{\epsilon_{k}}^{2}+\frac{1}{2} u_{\epsilon_{k}, x}^{2}+\frac{1}{2} \gamma_{\epsilon_{k}}^{2}-\frac{1}{2} \gamma_{\epsilon_{k}, x}^{2}\right) \rightarrow p *\left(u^{2}+\frac{1}{2} u_{x}^{2}+\frac{1}{2} \gamma^{2}-\frac{1}{2} \gamma_{x}^{2}\right), \\
& p *\left(u_{\epsilon_{k}, x} \gamma_{\epsilon_{k}, x}\right) \rightarrow p *\left(u_{x} \gamma_{x}\right),
\end{aligned}
$$

and

$$
p *\left(u_{\epsilon_{k}, x} \gamma_{\epsilon_{k}}\right) \rightarrow p *\left(u_{x} \gamma\right)
$$

in the sense of distributions on $\mathbb{R}_{+} \times \mathbb{R}$. This implies that $z$ is a global weak solution of (1.1). This completes the proof of Theorem 1.1.

\section{Acknowledgements}

This work was partially supported by NNSFC (No. 10971235), RFDP (No. 200805580014), NCET (No. NCET08-0579) and the key project of Sun Yat-sen University. The authors thank two referees for their valuable comments and suggestions.

\section{References}

[1] V.I. Arnold, Sur la géométrie différentielle des groupes de Lie de dimension infinie et ses applications à l'hydrodynamique es fluides parfaits, Ann. Inst. Fourier 16 (1966) 319-361.

[2] R. Beals, D. Scattinger, J. Szmigielski, Acoustic scatting and the extended Korteweg-de Vries hierarchy, Adv. Math. 140 (1998) 190-206.

[3] A. Bressan, A. Constantin, Global conservative solutions of the Camassa-Holm equation, Arch. Ration. Mech. Anal. 183 (2007) $215-239$.

[4] A. Bressan, A. Constantin, Global dissipative solutions of the Camassa-Holm equation, Anal. Appl. 5 (2007) 1-27.

[5] R. Camassa, D. Holm, An integrable shallow water equation with peaked solitons, Phys. Rev. Lett. 71 (1993) $1661-1664$.

[6] R. Camassa, D. Holm, J. Hyman, A new integrable shallow water equation, Adv. Appl. Mech. 31 (1994) 1-33.

[7] M. Chen, S.-Q. Liu, Y. Zhang, A 2-component generalization of the Camassa-Holm equation and its solutions, Lett. Math. Phys. 75 (2006) $1-15$.

[8] G.M. Coclite, H. Holden, K.H. Karlsen, Global weak solutions to a generalized hyperelastic-rod wave equation, SIAM J. Math. Anal. 37 (2006) 1044-1069.

[9] A. Constantin, The Hamiltonian structure of the Camassa-Holm equation, Expo. Math. 15 (1997) 53-85.

[10] A. Constantin, On the inverse spectral problem for the Camassa-Holm equation, J. Funct. Anal. 155 (1998) 352-363.

[11] A. Constantin, Existence of permanent and breaking waves for a shallow water equation: a geometric approach, Ann. Inst. Fourier (Grenoble) 50 (2000) 321-362.

[12] A. Constantin, On the scattering problem for the Camassa-Holm equation, Proc. R. Soc. Lond. Ser. A 457 (2001) $953-970$.

[13] A. Constantin, The trajectories of particles in Stokes waves, Invent. Math. 166 (2006) 523-535.

[14] A. Constantin, D. Lannes, The hydrodynamical relevance of the Camassa-Holm and Degasperis-Procesi equations, Arch. Ration. Mech. Anal. 193 (2009) 165-186.

[15] A. Constantin, J. Escher, Well-posedness, global existence and blowup phenomena for a periodic quasi-linear hyperbolic equation, Comm. Pure Appl. Math. 51 (1998) 475-504.

[16] A. Constantin, J. Escher, Wave breaking for nonlinear nonlocal shallow water equations, Acta. Math. 181 (1998) $229-243$.

[17] A. Constantin, J. Escher, On the blow-up rate and the blow-up of breaking waves for a shallow water equation, Math. Z. 233 (2000) $75-91$.

[18] A. Constantin, V. Gerdjikov, R. Ivanov, Inverse scattering transform for the Camassa-Holm equation, Inverse Problems 22 (2006) $2197-2207$.

[19] A. Constantin, J. Escher, Particle trajectories in solitary water waves, Bull. Amer. Math. Soc. 44 (2007) $423-431$.

[20] A. Constantin, R.S. Johnson, Propagation of very long water waves, with vorticity, over variable depth, with applications to tsunamis, Fluid Dynam. Res. 40 (2008) 175-211.

[21] A. Constantin, B. Kolev, Integrability of invariant metrics on the diffeomorphism group of the circle, J. Nonlinear Sci. 16 (2006) 109-122.

[22] A. Constantin, H.P. McKean, A shallow water equation on the circle, Comm. Pure Appl. Math. 52 (1999) 949-982.

[23] A. Constantin, L. Molinet, Global weak solutions for a shallow water equation, Comm. Math. Phys. 211 (2000) 45-61.

[24] A. Constantin, W. Strauss, Stability of peakons, Comm. Pure Appl. Math. 53 (2000) 603-610.

[25] A. Constantin, R.I. Ivanov, On an integrable two-component Camassa-Holm shallow water system, Phys. Lett. A 372 (2008) $7129-7132$.

[26] R. Curtain, H. Zwart, An Introduction to Infinite-Dimensional Linear Systems Theory, Springer-Verlag, New York, 1995.

[27] H. Dai, Model equations for nonlinear dispersive waves in compressible Mooney-Rivlin rod, Acta Mech. 127 (1998) $193-207$.

[28] R. Danchin, A few remarks on the Camassa-Holm equation, Differential Integral Equations 14 (2001) 953-988.

[29] R.J. DiPerna, P.L. Lions, Ordinary differential equations, transport theory and Sobolev space, Invent. Math. 98 (1989) $511-547$.

[30] P.G. Drazin, R.S. Johnson, Solitons: an Introduction, Cambridge University Press, Cambridge, 1989. 
[31] J. Escher, O. Lechtenfeld, Z. Yin, Well-posedness and blow-up phenomena for the 2-component Camassa-Holm equation, Discrete Contin. Dyn. Syst. 19 (2007) 493-513.

[32] A. Fokas, B. Fuchssteiner, Symplectic, their Bäcklund transformation and hereditary symmetries, Phys. D 4 (1981) 47-66.

[33] G. Grahovski, R.I. Ivanov, Generalized Fourier transform and perturbations to soliton equations, Discrete Contin. Dyn. Syst. Ser. B 12 (2009) 579-595.

[34] C. Guan, Z. Yin, Global existence and blow-up phenomena for an integrable two-component Camassa-Holm shallow water system, J. Differential Equations 248 (2010) 2003-2014.

[35] C. Guan, K.H. Karlsen, Z. Yin, Well-posedness and blow-up phenomena for a modified tow-component Camassa-Holm equation, in: Proceedings of the 2008-2009 Special Year in Nonlinear Partial Differential Equations, in: Contemp. Math., vol. 526, Amer. Math. Soc., 2010, pp. 199-220.

[36] H. Holden, X. Raynaud, Global conservative multipeakon solutions of the Camassa-Holm equation, J. Hyperbolic Differ. Equ. 4 (2007) 39-64.

[37] H. Holden, X. Raynaud, Global conservative solutions of the Camassa-Holm equation - A Lagrangian point of view, Comm. Partial Differential Equations 32 (2007) 1511-1549.

[38] H. Holden, X. Raynaud, Dissipative solutions for the Camassa-Holm equation, Discrete Contin. Dyn. Syst. 24 (2009) $1047-1112$.

[39] D. Holm, J. Marsden, T. Ratiu, The Euler-Poincaré equations and semidirect products with applications to continuum theories, Adv. Math. 137 (1998) 1-81.

[40] D. Holm, L. Naraigh, C. Tronci, Singular solution of a modified two-component Camassa-Holm equation, Phys. Rev. E 79 (2009) 1-13.

[41] I.P. Natanson, Theory of Functions of a Real Variable, vol. 1, Frederick Ungar Publishing Co., New York, 1974.

[42] J. Simon, Compact sets in the space $L^{p}(0, T ; B)$, Ann. Mat. Pura Appl. (4) 146 (1987) 65-96.

[43] T. Kato, Quasi-linear equations of evolution, with applications to partial differential equations, in: Spectral Theory and Differential Equations, in: Lecture Notes in Math., vol. 448, Springer-Verlag, Berlin, 1975, pp. 25-70.

[44] T. Kato, G. Ponce, Commutator estimates and Navier-Stokes equations, Comm. Pure Appl. Math. 41 (1988) 203-208.

[45] B. Kolev, Poisson brackets in hydrodynamics, Discrete Contin. Dyn. Syst. 19 (2007) 555-574.

[46] M. Lakshmanan, Integrable nonlinear wave equations and possible connections to tsunami dynamics, in: Tsunami and Nonlinear Waves, Springer, Berlin, 2007, pp. 31-49.

[47] Y. Li, P. Oliver, Well-posedness and blow-up solutions for an integrable nonlinear dispersive model wave equation, J. Differential Equations 162 (2000) 27-63.

[48] P.L. Lions, Mathematical Topics in Fluid Mechanics, vol. I. Incompressible Models, Oxford Lecture Ser. Math. Appl., vol. 3, Clarendon, Oxford University Press, New York, 1996.

[49] A. Pazy, Semigroup of Linear Operators and Applications to Partial Differential Equations, Springer-Verlag, New York, 1983.

[50] G.B. Whitham, Linear and Nonlinear Waves, J. Wiley and Sons, New York, 1980.

[51] Z. Xin, P. Zhang, On the weak solutions to a shallow water equation, Comm. Pure Appl. Math. 53 (2000) 1411-1433.

[52] Z. Yin, Well-posedness, global existence and blowup phenomena for an integrable shallow water equation, Discrete Contin. Dyn. Syst. 10 (2004) 393-411. 